\title{
DOUBLE PIERI ALGEBRAS AND ITERATED PIERI ALGEBRAS FOR THE CLASSICAL GROUPS
}

\author{
ROGER HOWE, SANGJIB KIM, AND SOO TECK LEE
}

\begin{abstract}
We study iterated Pieri rules for representations of classical groups. That is, we consider tensor products of a general representation with multiple factors of representations corresponding to one-rowed Young diagrams (or in the case of the general linear group, also the duals of these). We define iterated Pieri algebras, whose structure encodes the irreducible decompositions of such tensor products. We show that there is a single family of algebras, which we call double Pieri algebras, and which can be used to describe the iterated Pieri algebras for all three families of classical groups. Furthermore, we show that the double Pieri algebras have flat deformations to Hibi rings on explicitly described posets.
\end{abstract}

\section{INTRODUCTION}

1.1. The problem of computing tensor products of representations, motivated in part by the need to describe interaction of systems in quantum mechanics, has been the subject of a large literature. For a long time, the main focus was on the general linear group $\mathrm{GL}_{n}=\mathrm{GL}_{n}(\mathbb{C})$. For this group, the description of tensor product multiplicities is given by the LittlewoodRichardson rule ([LR]), which was fully justified only long after its original formulation, and for which many proofs have been given ([Sch, Th1, Th2, Ma, Stm, Ze, RS, HL2]). Recently, the techniques of quantum groups $([\mathrm{Kas}, \mathrm{Lu}])$ and Littelmann's path method $([\mathrm{Li}])$ have produced comparable results for a general complex reductive algebraic group/Lie algebra.

A particular case of tensor product decomposition for representations of $\mathrm{GL}_{n}$, when one of the factors is a symmetric power of the standard representation on $\mathbb{C}^{n}$, can be described by the Pieri rule, named after geometer Mario Pieri, which also describes the product of a Schubert cycle by a special Schubert cycle in the Schubert calculus. It has a simple description in the language of semistandard tableaux, and it is easy to iterate, producing descriptions of tensor products of the form

$$
\rho \otimes\left(\bigotimes_{j=1}^{\ell} \rho_{n}^{\left(a_{j}\right)}\right),
$$

where $\rho$ is any irreducible polynomial representation of $\mathrm{GL}_{n}$, and $\rho_{n}^{(a)}$ is the representation of $\mathrm{GL}_{n}$ on the $a$-th symmetric power of $\mathbb{C}^{n}$.

1.2. Although the Pieri rule is a very special case, it is of particular interest because one of the reciprocity laws associated to see-saw pairs of dual pairs $([\mathrm{Ku}])$ connects the Pieri rule with the branching rule from $\mathrm{GL}_{n}$ to $\mathrm{GL}_{n-1}$, and when this is iterated, one arrives

2010 Mathematics Subject Classification. 20G05, 05E15.

Key words and phrases. Tensor product, Pieri rule, Classical groups.

The third named author is partially supported by the MOE grant MOE2010-T2-2-113. 
at a description of a basis for each irreducible representation of $\mathrm{GL}_{n}$. This approach to describing representations of $\mathrm{GL}_{n}$ was explained by Gelfand and Tsetlin in 1950 ([GT]).

An alternative description of the representations of $\mathrm{GL}_{n}$ had been given somewhat earlier by Hodge $([\mathrm{Hd}])$, who worked in the flag algebra $\mathcal{R}\left(\mathrm{GL}_{n} / U_{n}\right)$ of regular functions on the homogeneous space $\mathrm{GL}_{n} / U_{n}$, where $U_{n}$ here denotes the group of unipotent upper triangular matrices - the maximal unipotent subgroup of $\mathrm{GL}_{n}$. (Actually, Hodge worked, not with the full algebra $\mathcal{R}\left(\mathrm{GL}_{n} / U_{n}\right)$, but with the subalgebra $\mathcal{R}^{+}\left(\mathrm{GL}_{n} / U_{n}\right)$ of "polynomial representations", whose matrix coefficients restricted to the diagonal torus are polynomial functions. Gelfand and Tsetlin also worked with polynomial representations.) This description works with a set of generators of $\mathcal{R}^{+}\left(\mathrm{GL}_{n} / U_{n}\right)$, and describes a family of monomials in these generators that constitutes a basis for $\mathcal{R}^{+}\left(\mathrm{GL}_{n} / U_{n}\right)$. These monomials are called "standard", so the description is called standard monomial theory.

For around 50 years, the Gelfand-Tsetlin description and the standard monomial description led more or less separate existences, but in the 1990s, they were connected by Gonciulea and Lakshmibai ([GL]), and by Sturmfels in a more restricted context ([Stu]). This advance was further refined in [KM], [MS] and [Ki1]. Gonciulea and Lakshmibai formulated their result in terms of a flat deformation of $\mathrm{GL}_{n} / U_{n}$ to a toric variety. Since their work, other authors have established that $G / U$, the "torus bundle over the flag variety", for any reductive algebraic group $G$, and even any multiplicity-free variety for $G$, has a flat toric deformation ([AB, Ca, Ch, DY, Kav $]$ ).

Although toric deformation gives insight into the nature of a variety, or at least its coordinate ring, it does not answer all questions about the variety or its coordinate ring. The coordinate ring of an affine toric variety is the semigroup ring of a lattice cone, by which we mean the intersection of $\mathbb{Z}^{n}$ with a rational polyhedral convex cone in $\mathbb{R}^{n}$, and there are many, many lattice cones in every dimension, and many unanswered questions about their geometry. For example, it was key for solving the Horn conjecture that the lattice cone attached to the Littlewood-Richardson rule should satisfy the "saturation property", and showing this was the main achievement of Knutson and Tao ([KT1]) (see also [Bu, KT2, Kly]).

1.3. Thus, it is of interest that the toric variety found by Gonciulea-Lakshmibai (and more explicitly by Kogan-Miller [KM] and Miller-Sturmfels [MS]), is particularly nice: it is the variety associated to the Gelfand-Tsetlin cone. Also, the associated semigroup ring is very nice: it is a Hibi ring. Hibi rings were defined by Hibi in the 1980s, in terms of generators and relations, as part of the effort to understand standard monomial theory in structural terms ([Hi] $)$. The generators of a Hibi ring are elements of a distributive lattice. It turns out, however, as was already noted by Hibi, that Hibi rings can be described more directly, as the semigroup rings of the lattice cone $\left(\mathbb{Z}^{+}\right)^{X, \geq}$ of non-negative integer-valued, order-preserving functions on a partially ordered set $X$ (whose partial order is $\geq$ ). The connection between Hibi's construction and the description as $\left(\mathbb{Z}^{+}\right)^{X, \geq}$ is mediated by Birkhoff's representation theorem for distributive lattices. The poset $\Gamma$ for which the Gelfand-Tsetlin cone is $\left(\mathbb{Z}^{+}\right)^{\Gamma, \geq}$ is the Gelfand-Tsetlin poset, whose elements consist of placeholders for entries in GelfandTsetlin patterns. To the best of our knowledge, the first explicit formulation of standard monomial theory in terms of $\left(\mathbb{Z}^{+}\right)^{\Gamma, \geq}$ was given by one of the present authors in his thesis in 2005 (see [Ki1]).

The $\left(\mathbb{Z}^{+}\right)^{X, \geq}$ are very special among lattice cones. In particular, it is evident that there are only finitely many of them in each dimension, among the infinite zoo of all lattice cones. 
Moreover, these cones have some very pleasant properties. Their semigroup ring has an explicitly describable set of generators and relations, with the relations being quadratic in the generators. Moreover, each cone $\left(\mathbb{Z}^{+}\right)^{X, \geq}$ has a canonical "triangulation", a decomposition into congruent integrally simplicial cones. This provides an explicit abstract version of Hodge's standard monomial theory for the semigroup rings of these cones.

Thus, it is natural to ask, are there other flag varieties besides $\mathrm{GL}_{n} / U_{n}$ that can be described in terms of Hibi rings, aka the lattice cones $\left(\mathbb{Z}^{+}\right)^{X, \geq}$ ? The answer is yes. It is easy to see that the coordinate rings attached to varieties of non-complete flags (nonminimal parabolics) for $\mathrm{GL}_{n}$ can also be described by posets derived from the GelfandTsetlin poset by fairly straightforward recipes. Also, starting from the other end, one can see that the branching algebras that describe the restriction of representations from $\mathrm{GL}_{n}$ to $\mathrm{GL}_{k} \times\left(\mathrm{GL}_{1}\right)^{n-k}$ likewise have deformations to Hibi rings, and with posets derivable from the Gelfand-Tsetlin poset.

More interestingly, [Ki1] showed that standard monomial theory for the symplectic group $\mathrm{Sp}_{2 n}$ could also be expressed as describing a toric deformation to the semigroup ring of $\left(\mathbb{Z}^{+}\right)^{\Gamma_{\mathrm{S}}, \geq}$, for an appropriate poset $\Gamma_{S p}([\mathrm{Kir}, \mathrm{Mo}, \mathrm{Zh}])$. Recently, Kim has shown that the branching algebra from $\mathrm{Sp}_{2 n}$ to $\mathrm{Sp}_{2 k}$ is a deformation of a Hibi ring, and in fact, isomorphic in certain ranges to an associated branching algebra for $\mathrm{GL}_{2 n}$. He has also shown that analogous branching algebras for orthogonal groups, from $\mathrm{SO}_{n}$ to $\mathrm{SO}_{k}$, subject to a suitable stability condition, are deformations of Hibi rings (see [Ki2, Ki4, KY]).

1.4. Returning to the Pieri rule as a decomposition of tensor products for $\mathrm{GL}_{n}$, since there is so much similarity in the branching rules and flag algebras for all the classical groups, one can ask about analogs of the Pieri rule for symplectic or orthogonal groups. Is there a simple description of tensor products $\tau \otimes \tau_{2 n}^{(a)}$, where $\tau$ is an irreducible representation of $\operatorname{Sp}_{2 n}$ and $\tau_{2 n}^{(a)}$ is the representation on the $a$-fold symmetric power of the standard representation on $\mathbb{C}^{2 n}$ ? The analogous question for orthogonal groups would concern tensor products $\sigma \otimes \sigma_{n}^{(a)}$, where $\sigma_{n}^{(a)}$ denotes the action of $\mathrm{SO}_{n}$ on the spherical harmonics of degree $a$ on $\mathbb{C}^{n}$.

Although the Pieri rule for $\mathrm{GL}_{n}$ is equivalent to the $\mathrm{GL}_{n}$ to $\mathrm{GL}_{n-1}$ branching rule, the analogs for $\mathrm{Sp}_{2 n}$ and $\mathrm{SO}_{n}$ are noticeably more complicated than the corresponding branching rules. There is a description of such tensor products in terms of tableaux ([Su1, Su2]), but it involves erasing boxes as well as adding boxes, making descriptions of iterated tensor products somewhat awkward. However, two of the present authors ([KL]) have found that an algebra naturally associated to the Pieri-like tensor products for $\mathrm{Sp}_{2 n}$ and $\mathrm{SO}_{n}$, is a deformation of a Hibi ring, at least under a certain stability condition. Furthermore, this description can easily be extended to iterated tensor products $\tau \otimes\left(\otimes_{j=1}^{\ell} \tau_{2 n}^{\left(a_{j}\right)}\right)$ and $\sigma \otimes\left(\bigotimes_{j=1}^{\ell} \sigma_{n}^{\left(a_{j}\right)}\right)$, and the resulting ring is again a deformation of a Hibi ring.

For the symplectic and orthogonal groups, the standard representation is self-dual, and this can be seen as a reason for the extra complexity of computing tensor products with the $\tau_{2 n}^{(a)}$ or $\sigma_{n}^{(a)}$. For the general linear group, of course the dual representation on $\left(\mathbb{C}^{n}\right)^{*}$ is distinct from the standard action on $\mathbb{C}^{n}$. However, by duality, there are parallels of the Pieri rule and the iterated Pieri rule also for tensoring with symmetric powers of $\left(\mathbb{C}^{n}\right)^{*}$. As far as we know, although iterations of the original Pieri rule have at least implicitly been used in describing branching, what happens when tensoring by the symmetric powers 
$\rho_{n}^{(a)}$ is combined with tensoring by dual symmetric powers $\left(\rho_{n}^{(b)}\right)^{*}$ has not been explicitly considered before. It was this question that motivated the present paper.

Our first result is that the ring associated to the combined multiple Pieri and dual Pieri rules is again a deformation of a Hibi ring, and we describe the associated poset explicitly. But the answer to this question has revealed a deeper unity than the authors suspected when starting this investigation. It turns out that there is a single (Hibi) ring in terms of which all the iterated Pieri algebras, for the symplectic and orthogonal groups, and the combined standard and dual Pieri algebras for the general linear group have a simple description. It is obtained by applying the iterated Pieri construction simultaneously to a product of general linear groups. For this reason, we call it the double Pieri algebra. Describing this algebra, and how it controls all the iterated Pieri algebras, is the main goal of the paper, described in Section 4.

Finally we remark that not only are Hibi lattice cones special among all lattice cones, the Hibi lattice cones that appear in representation theory are of a very restricted type. Our main result goes some distance toward explaining the notable family resemblance between the semigroups involved in the known representation-theoretic Hibi rings.

1.5. Thus, the role of Hibi rings in describing the representation theory of the classical groups is extensive. They do not, however, answer all questions. In collaboration with others, two of the authors showed that the (stable) branching algebras associated to classical symmetric pairs have toric deformations $\left(\left[\mathrm{HJL}^{+}\right]\right)$. The lattice cones involved in these deformations include the Littlewood-Richardson (LR) cone studied by Knutson and Tao ([KT1, KT2]) and others (see, for example, $[\mathrm{PV}]$ and $[\mathrm{DK}]$ ), and in fact, they can all be described in terms of the LR cone. These cones are substantially more complicated than the cones attached to posets.

On the other hand, there is a sense in which the LR cone is constructed from poset cones. See [HL2], which shows how the LR rule can be deduced from the Pieri rule by representation theoretic arguments. Thus, the geometric structure of cones appearing in representation theory presents some interesting questions, and the results of [HL2] suggest that poset cones and Hibi rings can play a useful role in understanding more complex cones and rings. In particular, one can ask if there is a way to understand the tensor product algebras for $\mathrm{Sp}_{2 n}$ and $\mathrm{SO}_{n}$ in terms of iterated Pieri algebras, analogous to the results of [HL2]. At the same time, it seems worthwhile to specify which representation theory problems can be described by Hibi rings. The present paper is a contribution to this project. It seems in fact that Hibi rings will help to provide a sharp description of the decompositions described in [Ho1] of polynomial rings as products of harmonics and invariants associated to dual pairs. This would in some sense provide reasonably complete picture of the classical actions considered by Weyl. The authors hope to study these questions further.

1.6. This paper is arranged as follows: In Section 2, we introduce notation for the representations of $\mathrm{GL}_{n}, \mathrm{O}_{n}$ and $\mathrm{Sp}_{2 n}$. In Section 3, we review the construction and the structure of an algebra, called the polynomial iterated Pieri algebra for $\mathrm{GL}_{n}$. This algebra encodes a polynomial version of the iterated Pieri rule for $\mathrm{GL}_{n}$. The results in this section are essentially a part of the much-studied standard monomial theory ([GL, Ho2, Ki1, MS]), originally due to Hodge $([\mathrm{Hd}])$ with refinements as noted above. In Section 4, we introduce the double Pieri algebra $\mathcal{L}_{(n, p),(k, q)}$ and study its structure. We define the general iterated Pieri algebra $\mathfrak{A}_{n, k, \ell, p, q}$ for $\mathrm{GL}_{n}$ in Section 5. We call this algebra stable when $k+p+\ell+q \leq n$. We show in Section 6 that when the algebra $\mathfrak{A}_{n, k, \ell, p, q}$ is stable, it can be expressed in terms 
of $\mathcal{L}_{(n, p),(k, q)}$ and a variant form $\widetilde{\mathcal{L}}_{(n, q),(\ell, p)}$. Finally in Section 7 , we describe the relation between the $\mathrm{O}_{n}$ (mutatis mutandem, $\mathrm{Sp}_{2 m}$ ) stable iterated Pieri algebra $\mathcal{A}_{n, k, p}$ defined in $[\mathrm{KL}]$ and $\mathcal{L}_{(n, p),(k, p)}$, and also between $\mathcal{A}_{n, k, p}$ and $\mathfrak{A}_{n, k, 0, p, p}$.

\section{Preliminaries}

In this section, we introduce notation for the irreducible representations of $\mathrm{GL}_{n}, \mathrm{O}_{n}$ and $\mathrm{Sp}_{2 n}$.

2.1. Representations of $\mathrm{GL}_{n}$. Let $\mathrm{GL}_{n}=\mathrm{GL}_{n}(\mathbb{C})$ denote the complex general linear group. Let $A_{n}$ be the diagonal torus in $\mathrm{GL}_{n}$ and let $U_{n}$ be the maximal unipotent subgroup consisting of all $n \times n$ upper triangular matrices with 1's on the diagonal. Then $B_{n}=A_{n} U_{n}$ is the standard Borel subgroup of $\mathrm{GL}_{n}$, and the irreducible rational representations of $\mathrm{GL}_{n}$ are in bijective correspondence with the semigroup $\widehat{A}_{n}^{+}$of dominant weights for $\mathrm{GL}_{n}$ with respect to $B_{n}$. For each $\alpha=\left(\alpha_{1}, \ldots, \alpha_{n}\right) \in \mathbb{Z}^{n}$, let us define

$$
\psi_{n}^{\alpha}: A_{n} \longrightarrow \mathbb{C}^{\times} \text {as } \psi_{n}^{\alpha}\left(\operatorname{diag}\left(a_{1}, a_{2}, \ldots, a_{n}\right)\right)=a_{1}^{\alpha_{1}} a_{2}^{\alpha_{2}} \cdots a_{n}^{\alpha_{n}}
$$

where $\operatorname{diag}\left(a_{1}, \ldots, a_{n}\right)$ denotes the diagonal matrix with diagonal entries $a_{1}, \ldots, a_{n}$. Then

$$
\widehat{A}_{n}^{+}=\left\{\psi_{n}^{\alpha}: \alpha=\left(\alpha_{1}, \ldots, \alpha_{n}\right) \in \mathbb{Z}^{n}, \alpha_{1} \geq \alpha_{2} \geq \cdots \geq \alpha_{n}\right\} .
$$

We shall denote by $\rho_{n}^{\alpha}$ the irreducible representation of $\mathrm{GL}_{n}$ with highest weight $\psi_{n}^{\alpha} \in \widehat{A}_{n}^{+}$.

We will also use diagram notation. Recall that a Young diagram $D$ is an array of square boxes arranged in left-justified horizontal rows, with each row no longer than the one above it $([\mathrm{Fu}])$. If $D$ has at most $r$ rows, then we shall denote it by

$$
D=\left(d_{1}, \ldots, d_{r}\right)
$$

where for each $i, d_{i}$ is the number of boxes in the $i$-th row of $D$. The depth of $D$, denoted by $r(D)$, is the number of nonzero rows in $D$. The number of all boxes in $D, \sum_{i>1} d_{i}$, will be denoted by $|D|$.

Suppose now $\psi_{n}^{\alpha} \in \widehat{A}_{n}^{+}$where $\alpha=\left(\alpha_{1}, \ldots, \alpha_{n}\right)$. We shall associate a pair of Young diagrams $(D, E)$ with $\alpha$ as follows: If $\alpha_{n} \geq 0$, then $D=\alpha$ and $E=(0)$. If $\alpha_{1}<0$, then $D=(0)$ and $E=\left(-\alpha_{n}, \ldots,-\alpha_{1}\right)$. If $\alpha$ is of the form

$$
\alpha=\left(d_{1}, d_{2}, \ldots, d_{r}, 0, \ldots, 0,-e_{s},-e_{s-1}, \ldots,-e_{1}\right),
$$

where $d_{1} \geq d_{2} \geq \cdots \geq d_{r}>0$ and $e_{1} \geq e_{2} \geq \cdots \geq e_{s}>0$, then $D=\left(d_{1}, d_{2}, \ldots, d_{r}\right)$ and $E=\left(e_{1}, e_{2}, \ldots, e_{s}\right)$. We shall write $\psi_{n}^{\alpha}$ as $\psi_{n}^{D, E}$ and write the representation $\rho_{n}^{\alpha}$ as $\rho_{n}^{D, E}$. In the case when $E=(0)$, we shall write $\psi_{n}^{D,(0)}$ and $\rho_{n}^{D,(0)}$ simply as $\psi_{n}^{D}$ and $\rho_{n}^{D}$, respectively. A representation of the form $\rho_{n}^{D}$ where $D$ is a Young diagram is called a polynomial representation of $\mathrm{GL}_{n}$. The contragredient representation of a representation $V$ of $\mathrm{GL}_{n}$ will be denoted by $V^{*}$. It is well known $([\mathrm{Ho} 2])$ that $\left(\rho_{n}^{D, E}\right)^{*} \simeq \rho_{n}^{E, D}$.

2.2. Representations of $\mathrm{O}_{n}$. Let $\mathrm{O}_{n}=\mathrm{O}_{n}(\mathbb{C})$ be the subgroup of $\mathrm{GL}_{n}$ which preserves the symmetric bilinear form

$$
\left\langle\left(\begin{array}{c}
u_{1}, \\
\vdots \\
u_{n}
\end{array}\right),\left(\begin{array}{c}
v_{1}, \\
\vdots \\
v_{n}
\end{array}\right)\right\rangle=\sum_{j=1}^{n} u_{j} v_{n-j+1}
$$


on $\mathbb{C}^{n}$. The finite dimensional irreducible representations of $\mathrm{O}_{n}$ are parameterized by Young diagrams $D$ such that the sum of the lengths of the first two columns of $D$ does not exceed $n$ ([Wy, GW, Ho2]). For such a Young diagram $D$, we shall denote the $\mathrm{O}_{n}$ representation associated with $D$ by $\sigma_{n}^{D}$. Specifically, $\sigma_{n}^{D}$ is the irreducible representation of $\mathrm{O}_{n}$ generated by the $\mathrm{GL}_{n}$ highest weight vector in $\rho_{n}^{D}$. See Section 3.6 of [Ho2] for more details.

Let $\mathrm{SO}_{n}$ denote the subgroup of $\mathrm{O}_{n}$ containing elements of $\mathrm{O}_{n}$ with determinant 1 , and let

$$
A_{\mathrm{SO}_{n}}=A_{n} \cap \mathrm{SO}_{n} \text { and } U_{\mathrm{SO}_{n}}=U_{n} \cap \mathrm{SO}_{n}
$$

Explicitly,

$$
A_{\mathrm{SO}_{n}}= \begin{cases}\left\{\operatorname{diag}\left(a_{1}, \ldots, a_{m}, a_{m}^{-1}, \ldots, a_{1}^{-1}\right): a_{1}, \ldots, a_{m} \in \mathbb{C}^{\times}\right\} & n=2 m \\ \left\{\operatorname{diag}\left(a_{1}, \ldots, a_{m}, 1, a_{m}^{-1}, \ldots, a_{1}^{-1}\right): a_{1}, \ldots, a_{m} \in \mathbb{C}^{\times}\right\} & n=2 m+1 .\end{cases}
$$

If $D$ is a Young diagram with depth $r(D) \neq n / 2$, then the restriction of $\sigma_{n}^{D}$ to $\mathrm{SO}_{n}$ is irreducible. Moreover, if $r(D)<n / 2$ and $\phi_{n}^{D}: A_{\mathrm{SO}_{n}} \rightarrow \mathbb{C}^{\times}$is the restriction of the character $\psi_{n}^{D}$, given in (2.1), to $A_{\mathrm{SO}_{n}}$, then as a $\mathrm{SO}_{n}$ module, $\sigma_{n}^{D}$ has highest weight $\phi_{n}^{D}$.

2.3. Representations of $\mathrm{Sp}_{2 n}$. Let $\mathrm{Sp}_{2 n}=\mathrm{Sp}_{2 n}(\mathbb{C})$ be the subgroup of $\mathrm{GL}_{2 n}$ which preserves the symplectic form $(.,$.$) on \mathbb{C}^{2 n}$ given by

$$
\left(\left(\begin{array}{c}
u_{1} \\
\vdots \\
u_{2 n}
\end{array}\right),\left(\begin{array}{c}
v_{1} \\
\vdots \\
v_{2 n}
\end{array}\right)\right)=\sum_{j=1}^{n}\left(u_{j} v_{2 n+1-j}-u_{2 n+1-j} v_{j}\right) .
$$

The diagonal torus $A_{\mathrm{Sp}_{2 n}}$ of $\mathrm{Sp}_{2 n}$ is isomorphic to $\left(\mathbb{C}^{\times}\right)^{n}$. The finite dimensional irreducible representations of $\mathrm{Sp}_{2 n}$ are parametrized by Young diagrams with at most $n$ rows. If $D$ is such a Young diagram, we shall denote the corresponding $\operatorname{Sp}_{2 n}$ representation by $\tau_{2 n}^{D}$. See Section 3.8 of [Ho2] for more details.

\section{Polynomial iterated Pieri algebras For $\mathrm{GL}_{n}$}

In this section, we review the construction and the structure of an algebra, called the polynomial iterated Pieri algebra for $\mathrm{GL}_{n}$. This algebra encodes a polynomial version of the iterated Pieri rule for $\mathrm{GL}_{n}$, and a description of its structure is essentially a part of the much-studied standard monomial theory ([GL, Ho2, Ki1, MS $]$ ), originally due to Hodge $([\mathrm{Hd}])$.

3.1. $\mathrm{GL}_{n}$ tensor product algebras. We first review an important result in Classical Invariant Theory which will be used in our later construction. Let $n$ and $m$ be positive integers, and let $\mathrm{M}_{n, m}=\mathrm{M}_{n, m}(\mathbb{C})$ denote the space of all $n \times m$ complex matrices. Let $\mathrm{GL}_{n} \times \mathrm{GL}_{m}$ act on $\mathrm{M}_{n, m}$ by

$$
\tau_{n, m}(g, h)(x)=\left(g^{-1}\right)^{t} x h^{-1}
$$

where $(g, h) \in \mathrm{GL}_{n} \times \mathrm{GL}_{m}$ and $x \in \mathrm{M}_{n, m}$. Here for a matrix $u, u^{t}$ denotes its transpose. Under the action $\tau_{n, m}$,

$$
\mathrm{M}_{n, m} \simeq \mathbb{C}^{n *} \otimes \mathbb{C}^{m *} .
$$

Here $\mathbb{C}^{n *}$ is the contragredient representation of the standard representation $\mathbb{C}^{n}$ of $\mathrm{GL}_{n}$, and similarly for $\mathbb{C}^{m *}$. The action $\tau_{n, m}$ induces the following action of $\mathrm{GL}_{n} \times \mathrm{GL}_{m}$ on the 
algebra $\mathcal{P}\left(\mathrm{M}_{n, m}\right)$ of polynomial functions on $\mathrm{M}_{n, m}$ (which will again be denoted by $\tau_{n, m}$ ): for $(g, h) \in \mathrm{GL}_{n} \times \mathrm{GL}_{m}, f \in \mathcal{P}\left(\mathrm{M}_{n, m}\right)$ and $x \in \mathrm{M}_{n, m}$,

$$
\left[\tau_{n, m}(g, h)(f)\right](x)=f\left(\tau_{n, m}\left(g^{-1}, h^{-1}\right)(x)\right)=f\left(g^{t} x h\right) .
$$

Theorem 3.1. ((GL $\left.\mathrm{GL}_{n}, \mathrm{GL}_{m}\right)$-duality, [Ho2, GW]) Under the action (3.2) by $\mathrm{GL}_{n} \times \mathrm{GL}_{m}$, $\mathcal{P}\left(\mathrm{M}_{n, m}\right)$ admits the following decomposition as a $\mathrm{GL}_{n} \times \mathrm{GL}_{m}$ module:

$$
\mathcal{P}\left(\mathrm{M}_{n, m}\right) \simeq \sum_{r(F) \leq \min (n, m)} \rho_{n}^{F} \otimes \rho_{m}^{F}
$$

Theorem 3.1 allows us to realize the irreducible representations of $\mathrm{GL}_{m}$ on a space of polynomial functions. More precisely, Let $\mathcal{P}\left(\mathrm{M}_{n, m}\right)^{U_{n}}$ be the algebra of polynomials in $\mathcal{P}\left(\mathrm{M}_{n, m}\right)$ which are invariant under the action by $U_{n}$. It is a module for $A_{n} \times \mathrm{GL}_{m}$, and is decomposed as

$$
\mathcal{P}\left(\mathrm{M}_{n, m}\right)^{U_{n}} \simeq \sum_{r(F) \leq \min (n, m)}\left(\rho_{n}^{F}\right)^{U_{n}} \otimes \rho_{m}^{F}
$$

For each Young diagram $F$ with $r(F) \leq \min (n, m)$, the space $\left(\rho_{n}^{F}\right)^{U_{n}}$ of vectors in $\rho_{n}^{F}$ invariant under $U_{n}$ is one-dimensional, and $A_{n}$ acts on it by the character $\psi_{n}^{F}$. So the subspace $\left(\rho_{n}^{F}\right)^{U_{n}} \otimes \rho_{m}^{F}$ of $\mathcal{P}\left(\mathrm{M}_{n, m}\right)^{U_{n}}$ is the eigenspace of $A_{n}$ in $\mathcal{P}\left(\mathrm{M}_{n, m}\right)^{U_{n}}$ corresponding to the eigencharacter $\psi_{n}^{F}$ and it is also a copy of the $\mathrm{GL}_{m}$ module $\rho_{m}^{F}$. It follows from equation (3.4) that the algebra $\mathcal{P}\left(\mathrm{M}_{n, m}\right)^{U_{n}}$ is a multiplicity free sum of irreducible representations of $\mathrm{GL}_{m}$.

Now suppose $k$ and $\ell$ are positive integers such that $m=k+\ell$. Then $\mathrm{GL}_{k} \times \mathrm{GL}_{\ell}$ embeds in $\mathrm{GL}_{m}$ as block diagonal matrices with blocks of size $k$ and $\ell$, so it acts on $\mathcal{P}\left(\mathrm{M}_{n, m}\right)$ by restriction. By extracting the $U_{k} \times U_{\ell}$ invariants in $\mathcal{P}\left(\mathrm{M}_{n, m}\right)^{U_{n}}$, we obtain

$$
\begin{aligned}
\mathcal{P}\left(\mathrm{M}_{n, m}\right)^{U_{n} \times U_{k} \times U_{\ell}} & =\left(\mathcal{P}\left(\mathrm{M}_{n, m}\right)^{U_{n}}\right)^{U_{k} \times U_{\ell}} \\
& \simeq \sum_{r(F) \leq \min (n, m)}\left(\rho_{n}^{F}\right)^{U_{n}} \otimes\left(\rho_{m}^{F}\right)^{U_{k} \times U_{\ell}}
\end{aligned}
$$

The algebra $\mathcal{P}\left(\mathrm{M}_{n, m}\right)^{U_{n} \times U_{k} \times U_{\ell}}$ is a module for $A_{n} \times A_{k} \times A_{\ell}$. For fixed Young diagrams $D, E$ and $F$, the $\psi_{n}^{F} \times \psi_{k}^{D} \times \psi_{\ell}^{E}$ eigenspace of $A_{n} \times A_{k} \times A_{\ell}$ in $\mathcal{P}\left(\mathrm{M}_{n, m}\right)^{U_{n} \times U_{k} \times U_{\ell}}$ can be identified with the space of $\mathrm{GL}_{k} \times \mathrm{GL}_{\ell}$ highest weight vectors in $\rho_{m}^{F}$ of weight $\psi_{k}^{D} \times \psi_{\ell}^{E}$, so its dimension gives the multiplicity of $\rho_{k}^{D} \otimes \rho_{\ell}^{E}$ in $\rho_{m}^{F}$. Thus the algebra structure of $\mathcal{P}\left(\mathrm{M}_{n, m}\right)^{U_{n} \times U_{k} \times U_{\ell}}$ encodes the branching rule from $\mathrm{GL}_{m}$ to $\mathrm{GL}_{k} \times \mathrm{GL}_{\ell}$. In view of this property, we call $\mathcal{P}\left(\mathrm{M}_{n, m}\right)^{U_{n} \times U_{k} \times U_{\ell}}$ a $\left(\mathrm{GL}_{m}, \mathrm{GL}_{k} \times \mathrm{GL}_{\ell}\right)$ branching algebra.

On the other hand, we have an isomorphism of algebras

$$
\mathcal{P}\left(\mathrm{M}_{n, m}\right) \simeq \mathcal{P}\left(\mathrm{M}_{n, k} \oplus \mathrm{M}_{n, \ell}\right) \simeq \mathcal{P}\left(\mathrm{M}_{n, k}\right) \otimes \mathcal{P}\left(\mathrm{M}_{n, \ell}\right)
$$


So $\left(\mathrm{GL}_{n} \times \mathrm{GL}_{k}\right) \times\left(\mathrm{GL}_{n} \times \mathrm{GL}_{\ell}\right) \simeq\left(\mathrm{GL}_{n} \times \mathrm{GL}_{n}\right) \times \mathrm{GL}_{k} \times \mathrm{GL}_{\ell}$ acts on $\mathcal{P}\left(\mathrm{M}_{n, m}\right)$ by $\tau_{n, k} \times \tau_{n, \ell}$. By Theorem 3.1, $\mathcal{P}\left(\mathrm{M}_{n, m}\right)$ is decomposed as

$$
\begin{aligned}
\mathcal{P}\left(\mathrm{M}_{n, m}\right) & \simeq\left(\sum_{r(D) \leq \min (n, k)} \rho_{n}^{D} \otimes \rho_{k}^{D}\right) \otimes\left(\sum_{r(E) \leq \min (n, \ell)} \rho_{n}^{E} \otimes \rho_{\ell}^{E}\right) \\
& \simeq \sum_{\substack{r(D) \leq \min (n, k) \\
r(E) \leq \min (n, \ell)}}\left(\rho_{n}^{D} \otimes \rho_{n}^{E}\right) \otimes \rho_{k}^{D} \otimes \rho_{\ell}^{E} .
\end{aligned}
$$

We now restrict the action of $\left(\mathrm{GL}_{n} \times \mathrm{GL}_{n}\right) \times \mathrm{GL}_{k} \times \mathrm{GL}_{\ell}$ on $\mathcal{P}\left(\mathrm{M}_{n, m}\right)$ to $\Delta\left(\mathrm{GL}_{n}\right) \times \mathrm{GL}_{k} \times \mathrm{GL}_{\ell}$ where $\Delta\left(\mathrm{GL}_{n}\right) \cong \mathrm{GL}_{n}$ embeds diagonally in $\mathrm{GL}_{n} \times \mathrm{GL}_{n}$ with $g \rightarrow(g, g)$. By extracting the $U_{k} \times U_{\ell}$ invariants in $\mathcal{P}\left(\mathrm{M}_{n, m}\right)$, we obtain

$$
\mathcal{P}\left(\mathrm{M}_{n, m}\right)^{U_{k} \times U_{\ell}} \simeq \sum_{\substack{r(D) \leq \min (n, k) \\ r(E) \leq \min (n, \ell)}}\left(\rho_{n}^{D} \otimes \rho_{n}^{E}\right) \otimes\left(\rho_{k}^{D}\right)^{U_{k}} \otimes\left(\rho_{\ell}^{E}\right)^{U_{\ell}} .
$$

The algebra $\mathcal{P}\left(\mathrm{M}_{n, m}\right)^{U_{k} \times U_{\ell}}$ is a module for $\mathrm{GL}_{n} \times A_{k} \times A_{\ell}$. For each pair $(D, E)$ of Young diagrams, the subspace $\left(\rho_{n}^{D} \otimes \rho_{n}^{E}\right) \otimes\left(\rho_{k}^{D}\right)^{U_{k}} \otimes\left(\rho_{\ell}^{E}\right)^{U_{\ell}}$ of $\mathcal{P}\left(\mathrm{M}_{n, m}\right)^{U_{k} \times U_{\ell}}$ is the eigenspace of $A_{k} \times A_{\ell}$ corresponding to the eigencharacter $\psi_{k}^{D} \times \psi_{\ell}^{E}$, and it is also a copy of the tensor product $\rho_{n}^{D} \otimes \rho_{n}^{E}$ of $\mathrm{GL}_{n}$ representations. By equation (3.5),

$$
\begin{aligned}
\mathcal{P}\left(\mathrm{M}_{n, m}\right)^{U_{n} \times U_{k} \times U_{\ell}} & =\left(\mathcal{P}\left(\mathrm{M}_{n, m}\right)^{U_{k} \times U_{\ell}}\right)^{U_{n}} \\
& \simeq \sum_{\substack{r(D) \leq \min (n, k) \\
r(E) \leq \min (n, \ell)}}\left(\rho_{n}^{D} \otimes \rho_{n}^{E}\right)^{U_{n}} \otimes\left(\rho_{k}^{D}\right)^{U_{k}} \otimes\left(\rho_{\ell}^{E}\right)^{U_{\ell}} .
\end{aligned}
$$

So the algebra $\mathcal{P}\left(\mathrm{M}_{n, m}\right)^{U_{n} \times U_{k} \times U_{\ell}}$ describes how tensor products of $\mathrm{GL}_{n}$ representations decompose. In view of this property, we also call $\mathcal{P}\left(\mathrm{M}_{n, m}\right)^{U_{n} \times U_{k} \times U_{\ell}}$ a $\mathrm{GL}_{n}$ tensor product algebra. Since the algebra $\mathcal{P}\left(\mathrm{M}_{n, m}\right)^{U_{n} \times U_{k} \times U_{\ell}}$ encodes two sets of branching information, it is a reciprocity algebra in the sense of [HTW1].

This construction can be generalized. If $m=k_{1}+k_{2}+\cdots+k_{p}$ where $k_{1}, \ldots, k_{p}$ are positive integers, then $\prod_{i=1}^{p} \mathrm{GL}_{k_{i}}$ embeds diagonally in $\mathrm{GL}_{m}$ and the algebra

$$
\mathcal{P}\left(\mathrm{M}_{n, m}\right)^{U_{n} \times\left(\prod_{i=1}^{p} U_{k_{i}}\right)}
$$

describes the branching rule from $\mathrm{GL}_{m}$ to $\prod_{i=1}^{p} \mathrm{GL}_{k_{i}}$ as well as the decomposition of tensor products of $p$ representations of $\mathrm{GL}_{n}$. Readers may refer to [HL1] for more details.

3.2. Polynomial Pieri algebras for $\mathrm{GL}_{n}$. Let $m=k+\ell$. We shall write a typical element of $\mathrm{M}_{n, m}$ as

$$
\left(\begin{array}{cccccccc}
x_{11} & x_{12} & \cdots & x_{1 k} & y_{11} & y_{12} & \cdots & y_{1 \ell} \\
x_{21} & x_{22} & \cdots & x_{2 k} & y_{21} & y_{22} & \cdots & y_{2 \ell} \\
\vdots & \vdots & & \vdots & \vdots & \vdots & & \vdots \\
x_{n 1} & x_{n 2} & \cdots & x_{n k} & y_{n 1} & y_{n 2} & \cdots & y_{n \ell}
\end{array}\right)
$$

so that $\mathcal{P}\left(\mathrm{M}_{n, m}\right)$ can be viewed as a polynomial algebra on the variables $x_{a b}$ and $y_{c d}$. In this subsection, we focus on the case when $\ell=1$. Since $U_{\ell}=U_{1}$ is trivial, by equation 
(3.6), we have

$$
\begin{aligned}
\mathcal{P}\left(\mathrm{M}_{n, k+1}\right)^{U_{n} \times U_{k}} & =\mathcal{P}\left(\mathrm{M}_{n, k+1}\right)^{U_{n} \times U_{k} \times U_{1}} \\
& \simeq \sum_{\substack{r(D) \leq \min (n, k) \\
a \in \mathbb{Z}^{+}}}\left(\rho_{n}^{D} \otimes \rho_{n}^{(a)}\right)^{U_{n}} \otimes\left(\rho_{k}^{D}\right)^{U_{k}} \otimes \rho_{1}^{(a)},
\end{aligned}
$$

where $\mathbb{Z}^{+}$denotes the set of all nonnegative integers. So the algebra $\mathcal{P}\left(\mathrm{M}_{n, k+1}\right)^{U_{n} \times U_{k}}$ describes tensor products of the form $\rho_{n}^{D} \otimes \rho_{n}^{(a)}$, where $(a)$ is the Young diagram with only 1 row and $a$ boxes, and $\rho_{n}^{(a)} \simeq S^{a}\left(\mathbb{C}^{n}\right)$ is the $a$-th symmetric power of $\mathbb{C}^{n}$. It is shown in [Ho2] that $\mathcal{P}\left(\mathrm{M}_{n, k+1}\right)^{U_{n} \times U_{k}}$ is a polynomial algebra on the free generators $\delta_{j}$ and $\delta_{(j-1,1)}$ where

$$
\delta_{i}=\left|\begin{array}{cccc}
x_{11} & x_{12} & \cdots & x_{1 i} \\
x_{21} & x_{22} & \cdots & x_{2 i} \\
\vdots & \vdots & & \vdots \\
x_{i 1} & x_{i 2} & \cdots & x_{i i}
\end{array}\right|, \quad 1 \leq i \leq \min (n, k)
$$

and

$$
\delta_{(j-1,1)}=\left|\begin{array}{ccccc}
x_{11} & x_{12} & \cdots & x_{1, j-1} & y_{11} \\
x_{21} & x_{22} & \cdots & x_{2, j-1} & y_{21} \\
\vdots & \vdots & & \vdots & \vdots \\
x_{j 1} & x_{j 2} & \cdots & x_{j, j-1} & y_{j 1}
\end{array}\right|, \quad 1 \leq j \leq \min (n, k+1)
$$

It follows that the set of all monomials in the $\delta_{j}$ and $\delta_{(j-1,1)}$ forms a basis for $\mathcal{P}\left(\mathrm{M}_{n, k+1}\right)^{U_{n} \times U_{k}}$. Using this fact, one can deduce the Pieri rule for $\mathrm{GL}_{n}$ (see [Ho2]).

Pieri rule for $\mathrm{GL}_{n}$. Let $D=\left(d_{1}, \ldots, d_{n}\right)$ be a Young diagram with at most $n$ rows and let $\alpha$ be a nonnegative integer. Then

$$
\rho_{n}^{D} \otimes \rho_{n}^{(\alpha)}=\sum_{F} \rho_{n}^{F}
$$

where the sum is taken over all Young diagrams $F=\left(f_{1}, \ldots, f_{n}\right)$ with at most $n$ rows such that $D \sqsubseteq F$ and $|F|-|D|=\alpha$. Here $D \sqsubseteq F$ means that

$$
f_{1} \geq d_{1} \geq f_{2} \geq d_{2} \geq \cdots \geq f_{n} \geq d_{n}
$$

The condition (3.7) is called the interlacing condition. Since the algebra $\mathcal{P}\left(\mathrm{M}_{n, k+1}\right)^{U_{n} \times U_{k}}$ encodes the Pieri rule, we will call it a (polynomial) Pieri algebra for $\mathrm{GL}_{n}$.

3.3. Polynomial iterated Pieri algebra for $\mathrm{GL}_{n}$. The construction in Section 3.2 can be generalized as follows. Let $m=k+\ell$, so that $\mathrm{GL}_{k} \times A_{\ell} \subseteq \mathrm{GL}_{k} \times \mathrm{GL}_{\ell} \subseteq \mathrm{GL}_{m}$. We consider the action $\tau_{n, m}$ of $\mathrm{GL}_{n} \times \mathrm{GL}_{m}$ on $\mathcal{P}\left(\mathrm{M}_{n, m}\right)$ defined in equation (3.2) and restrict 
it to $\mathrm{GL}_{n} \times\left(\mathrm{GL}_{k} \times A_{\ell}\right) \simeq \mathrm{GL}_{n} \times\left(\mathrm{GL}_{k} \times \mathrm{GL}_{1}^{\ell}\right)$. Then under this action,

$$
\begin{aligned}
& \mathcal{P}\left(\mathrm{M}_{n, m}\right) \simeq \mathcal{P}(\mathrm{M}_{n, k} \oplus \overbrace{\mathbb{C}^{n} \oplus \cdots \oplus \mathbb{C}^{n}}^{\ell} \simeq \mathcal{P}\left(\mathrm{M}_{n, k}\right) \otimes \overbrace{\mathcal{P}\left(\mathbb{C}^{n}\right) \otimes \cdots \otimes \mathcal{P}\left(\mathbb{C}^{n}\right)}^{\ell} \\
& \simeq\left(\sum_{r(D) \leq \min (n, k)} \rho_{n}^{D} \otimes \rho_{k}^{D}\right) \otimes\left(\sum_{\alpha_{1} \geq 0} \rho_{n}^{\left(\alpha_{1}\right)} \otimes \rho_{1}^{\left(\alpha_{1}\right)}\right) \otimes \cdots \otimes\left(\sum_{\alpha_{\ell} \geq 0} \rho_{n}^{\left(\alpha_{\ell}\right)} \otimes \rho_{1}^{\left(\alpha_{\ell}\right)}\right) \\
& \simeq \sum_{\substack{r(D) \leq \min (n, k) \\
\alpha_{1}, \ldots, \alpha_{\ell} \geq 0}}\left(\rho_{n}^{D} \otimes \rho_{n}^{\left(\alpha_{1}\right)} \otimes \cdots \otimes \rho_{n}^{\left(\alpha_{\ell}\right)}\right) \otimes \rho_{k}^{D} \otimes \rho_{1}^{\left(\alpha_{1}\right)} \otimes \cdots \otimes \rho_{1}^{\left(\alpha_{\ell}\right)} \\
& \simeq \sum_{\substack{r(D) \leq \min (n, k) \\
\alpha=\left(\alpha_{1}, \ldots, \alpha_{\ell}\right) \in\left(\mathbb{Z}^{+}\right)^{\ell}}}\left(\rho_{n}^{D} \otimes \rho_{n}^{\left(\alpha_{1}\right)} \otimes \cdots \otimes \rho_{n}^{\left(\alpha_{\ell}\right)}\right) \otimes \rho_{k}^{D} \otimes \psi_{\ell}^{\alpha}
\end{aligned}
$$

where the representation $\rho_{1}^{\left(\alpha_{1}\right)} \otimes \cdots \otimes \rho_{1}^{\left(\alpha_{\ell}\right)}$ of $\mathrm{GL}_{1}^{\ell}$ is identified with the character $\psi_{\ell}^{\alpha}$ of $A_{\ell}$. By extracting the $U_{n} \times U_{k}$ invariants from $\mathcal{P}\left(\mathrm{M}_{n, m}\right)$, we obtain

$$
\mathcal{P}\left(\mathrm{M}_{n, m}\right)^{U_{n} \times U_{k}} \simeq \sum_{\substack{r(D) \leq \min (n, k) \\ \alpha=\left(\alpha_{1}, \ldots, \alpha_{\ell}\right) \in\left(\mathbb{Z}^{+}\right)^{\ell}}}\left(\rho_{n}^{D} \otimes \rho_{n}^{\left(\alpha_{1}\right)} \otimes \cdots \otimes \rho_{n}^{\left(\alpha_{\ell}\right)}\right)^{U_{n}} \otimes\left(\rho_{k}^{D}\right)^{U_{k}} \otimes \psi_{\ell}^{\alpha} .
$$

Thus the algebra $\mathcal{P}\left(\mathrm{M}_{n, m}\right)^{U_{n} \times U_{k}}$ describes how tensor products of the form

$$
\rho_{n}^{D} \otimes \rho_{n}^{\left(\alpha_{1}\right)} \otimes \cdots \otimes \rho_{n}^{\left(\alpha_{\ell}\right)}
$$

decompose into irreducibles. In view of this property, we call $\mathcal{P}\left(\mathrm{M}_{n, m}\right)^{U_{n} \times U_{k}}$ a polynomial iterated Pieri algebra for $\mathrm{GL}_{n}$. It is a module for $A_{n} \times A_{k} \times A_{\ell}$, so it is graded and can be decomposed as

$$
\mathcal{P}\left(\mathrm{M}_{n, m}\right)^{U_{n} \times U_{k}}=\sum_{F, D, \alpha} W_{F, D, \alpha},
$$

where the sum is taken over all Young diagrams $D$ and $F$ with $r(D) \leq \min (n, k)$ and $r(F) \leq \min (n, m)$, and all $\alpha \in\left(\mathbb{Z}^{+}\right)^{\ell}$, and $W_{F, D, \alpha}$ is the $\psi_{n}^{F} \times \psi_{k}^{D} \times \psi_{\ell}^{\alpha}$-eigenspace of $A_{n} \times A_{k} \times A_{\ell}$. The dimension of $W_{F, D, \alpha}$ coincides with the multiplicity of $\rho_{n}^{F}$ in the tensor product (3.9), which can be obtained by applying the Pieri rule repeatedly.

Polynomial iterated Pieri rule for $\mathrm{GL}_{n}$. Let $D$ and $F$ be Young diagrams with at most $n$ rows and $\alpha=\left(\alpha_{1}, \ldots, \alpha_{\ell}\right) \in\left(\mathbb{Z}^{+}\right)^{\ell}$. Then the multiplicity of $\rho_{n}^{F}$ in the tensor product $\rho_{n}^{D} \otimes \rho_{n}^{\left(\alpha_{1}\right)} \otimes \cdots \otimes \rho_{n}^{\left(\alpha_{\ell}\right)}$ is given by the number of sequences of Young diagrams $\left(F_{0}, F_{1}, \ldots, F_{\ell}\right)$ such that

$$
D=F_{0} \sqsubseteq F_{1} \sqsubseteq \cdots \sqsubseteq F_{\ell-1} \sqsubseteq F_{\ell}=F,
$$

and

$$
\left|F_{j}\right|-\left|F_{j-1}\right|=\alpha_{j} \quad \text { for } 1 \leq j \leq \ell
$$

There is another description of this multiplicity. If a Young diagram $D$ sits inside another Young diagram $F$, then we write $D \subseteq F$. In this case, by removing all boxes belonging to $D$, we obtain the skew diagram $F / D$. If we put a positive number in each box of $F / D$, then it becomes a skew tableau and we say that the shape of this skew tableau is $F / D$. If the entries of this skew tableau are taken from $\{1,2, \ldots, \ell\}$, and $\alpha_{j}$ of them are $j$ for $1 \leq j \leq \ell$, then we say the content of this skew tableau is $\alpha=\left(\alpha_{1}, \ldots, \alpha_{\ell}\right)$. A skew tableau $T$ is called 
semistandard if the numbers in each row of $T$ weakly increase from left-to-right, and the numbers in each column of $T$ strictly increase from top-to-bottom.

\section{Notation.}

(i) Let $\mathrm{ST}_{(F, D, \alpha)}$ be the set of semistandard tableaux of shape $F / D$ and with content $\alpha$.

(ii) The number of elements in $\mathrm{ST}_{(F, D, \alpha)}$ is denoted by $K_{F / D, \alpha}$ and it is called a skew Kostka number ([Sta]).

(iii) We also let

$$
\mathrm{ST}_{(n, k, \ell)}=\bigcup_{F, D, \alpha} \mathrm{ST}_{(F, D, \alpha)}
$$

where the union is taken over all Young diagrams $F$ and $D$ with $r(F) \leq \min (n, k+\ell)$ and $r(D) \leq \min (n, k)$, and all $\alpha \in\left(\mathbb{Z}^{+}\right)^{\ell}$.

We now let $\left(F_{0}, F_{1}, \ldots, F_{\ell}\right)$ be a sequence of Young diagrams which satisfies conditions (3.11) and (3.12). We regard $F / D$ as a union of the skew diagrams $F_{i} / F_{i-1}, 1 \leq i \leq \ell$. By filling the boxes in $F_{i} / F_{i-1}$ with $i$ for each $1 \leq i \leq \ell$, we obtain a semistandard tableau $T$ of shape $F / D$ and content $\alpha=\left(\alpha_{1}, \ldots, \alpha_{\ell}\right)$. In fact,

$$
\left(F_{0}, F_{1}, \ldots, F_{\ell}\right) \longrightarrow T
$$

is a bijection between sequences of Young diagrams $\left(F_{0}, F_{1}, \ldots, F_{\ell}\right)$ which satisfy conditions (3.11) and (3.12), and semistandard tableaux $T$ in $\mathrm{ST}_{(F, D, \alpha)}$. It follows that

$$
\rho_{n}^{D} \otimes \rho_{n}^{\left(\alpha_{1}\right)} \otimes \cdots \otimes \rho_{n}^{\left(\alpha_{\ell}\right)}=\sum_{F} K_{F / D, \alpha} \rho_{n}^{F}
$$

and this implies the following lemma.

Lemma 3.2. The dimension of the homogeneous component $W_{F, D, \alpha}$ of $\mathcal{P}\left(\mathrm{M}_{n, m}\right)^{U_{n} \times U_{k}}$ is given by

$$
\operatorname{dim} W_{F, D, \alpha}=K_{F / D, \alpha}
$$

The lemma suggests that elements of $\mathrm{ST}_{F, D, \alpha}$ can be used to label a basis $\mathcal{B}_{F, D, \alpha}$ for $W_{F, D, \alpha}$ and the union of all $\mathcal{B}_{F, D, \alpha}$ will be a basis of the algebra $\mathcal{P}\left(\mathrm{M}_{n, m}\right)^{U_{n} \times U_{k}}$ labeled by elements of $\mathrm{ST}_{n, k, \ell}$.

3.4. SAGBI theory. In this subsection, we will recall the notion of a monomial order and some general results in $[\mathrm{CHV}]$. Let $\mathbb{C}\left[x_{1}, \ldots, x_{n}\right]$ be a complex polynomial algebra on the variables $x_{1}, \ldots, x_{n}$. A monomial in $\mathbb{C}\left[x_{1}, \ldots, x_{n}\right]$ is an element of the form

$$
x^{\alpha}=x_{1}^{\alpha_{1}} x_{2}^{\alpha_{2}} \cdots x_{n}^{\alpha_{n}}
$$

where $\alpha=\left(\alpha_{1}, \ldots, \alpha_{n}\right) \in\left(\mathbb{Z}^{+}\right)^{n}$. A monomial order is a total ordering $<$ on the set of monomials in $\mathbb{C}\left[x_{1}, \ldots, x_{n}\right]$, or equivalently on $\left(\mathbb{Z}^{+}\right)^{n}$, such that

(MO1) $(0,0, \ldots, 0)$ is the minimal element in $\left(\mathbb{Z}^{+}\right)^{n}$.

(MO2) Any decreasing sequence is finite.

(MO3) If $\alpha, \beta, \gamma \in\left(\mathbb{Z}^{+}\right)^{n}$ and $\alpha<\beta$, then $\alpha+\gamma<\beta+\gamma$. 
There are many examples of monomial orders on $\left(\mathbb{Z}^{+}\right)^{n}$ (see [CLO]). Here we will describe one which we will use later. We first choose a total ordering on the variables $x_{1}, \ldots, x_{n}$. Specifically, we choose a permutation $\sigma$ on $\{1,2, \ldots, n\}$ and declare that

$$
x_{\sigma(1)}>x_{\sigma(2)}>\cdots>x_{\sigma(n)} .
$$

We then extend this ordering to a total ordering on all monomials as follows: Given two monomials $x^{\alpha}$ and $x^{\beta}$, we have

$$
x^{\alpha}>x^{\beta}
$$

if either

(i) the total degree of $x^{\alpha}$ is higher than that of $x^{\beta}$; or

(ii) both $x^{\alpha}$ and $x^{\beta}$ have the same total degree, and for the first (i.e. largest) variable with an exponent in $x^{\alpha}$ that is different from its exponent in $x^{\beta}$, the exponent in $x^{\alpha}$ is larger.

We call this monomial order the graded lexicographic order with respect to (3.16) ([CLO]).

We now fix a monomial order on $\left(\mathbb{Z}^{+}\right)^{n}$. Let $f$ be a polynomial in $\mathbb{C}\left[x_{1}, \ldots, x_{n}\right]$. Since $f$ is a finite linear combination of the monomials $x^{\alpha}$, and since the monomial order is a total ordering, among the monomials appearing in $f$ with non-zero coefficients, there will be a maximal one. We call this the leading monomial of $f$, and denote it by $\operatorname{LM}(f)$. Then it follows from (MO3) that for $f, g \in \mathbb{C}\left[x_{1}, \ldots, x_{n}\right]$,

$$
\operatorname{LM}(f g)=\operatorname{LM}(f) \operatorname{LM}(g) .
$$

Let $R$ be a subalgebra of $\mathbb{C}\left[x_{1}, \ldots, x_{n}\right]$, and let

$$
\operatorname{LM}(R)=\{\operatorname{LM}(f): f \in R\} .
$$

Then by (3.17), $\operatorname{LM}(R)$ is a semigroup. The initial algebra of $R$, denoted by $\mathbb{C}[\operatorname{LM}(R)]$, is the subalgebra of $\mathbb{C}\left[x_{1}, \ldots, x_{n}\right]$ generated by $\operatorname{LM}(R)$. If the semigroup $\operatorname{LM}(R)$ is finitely generated, then a general result $([\mathrm{CHV}])$ says that $\mathbb{C}[\mathrm{LM}(R)]$ is a good approximation to $R$, in the following sense.

Theorem 3.3. Let $R$ be a subalgebra of a polynomial algebra $\mathbb{C}\left[x_{1}, \ldots, x_{n}\right]$. If the semigroup $\operatorname{LM}(R)$ is finitely generated, then there exists a flat one-parameter family of $\mathbb{C}$-algebras with general fibre $R$ and special fibre $\mathbb{C}[\operatorname{LM}(R)]$.

We will show later that in the case when $R=\mathcal{P}\left(\mathrm{M}_{n, m}\right)^{U_{n} \times U_{k}}$ as well as other algebras for which we are interested in, $\operatorname{LM}(R)$ is of a very special type and is indeed finitely generated. We define these semigroups in the next subsection.

3.5. Hibi cones. For a linear functional $\lambda$ on $\mathbb{R}^{n}$, the set

$$
H_{\lambda}^{+}=\left\{\mathbf{v} \in \mathbb{R}^{n}: \lambda(\mathbf{v}) \geq 0\right\}
$$

is called the closed half-space for $\lambda$. If $L=\left\{\lambda_{1}, \ldots, \lambda_{r}\right\}$ is a collection of linear functionals on $\mathbb{R}^{n}$, then the intersection

$$
C_{L}^{+}=\bigcap_{i=1}^{r} H_{\lambda_{i}}^{+}
$$

is called a convex polyhedral cone. It is called rational if each $\lambda_{i}$ in $L$ takes rational values on $\mathbb{Z}^{n}$. A lattice cone is the intersection of a rational convex polyhedral cone in $\mathbb{R}^{n}$ with $\mathbb{Z}^{n}$. It has a structure of an affine semigroup $([\mathrm{BH}])$, that is, it is a finitely generated subsemigroup of $\mathbb{Z}^{n}$ containing 0 . 
We now describe an important class of lattice cones which are called Hibi cones ([Hi, Ho3, Rei]). Let $\Gamma=\left\{\gamma_{1}, \ldots, \gamma_{N}\right\}$ be a finite poset with a partial ordering $\succeq$. We call a real-valued function $f$ on $\Gamma$ order preserving if

$$
x, y \in \Gamma, x \succeq y \Longrightarrow f(x) \geq f(y) .
$$

Let $\mathbb{R}^{+}$be the set of nonnegative real numbers and let

$$
\left(\mathbb{R}^{+}\right)^{\Gamma, \succeq}=\left\{f: \Gamma \rightarrow \mathbb{R}^{+}: f \text { is order preserving }\right\} .
$$

We shall identify each function $f: \Gamma \rightarrow \mathbb{R}$ with the point

$$
\left(f\left(\gamma_{1}\right), f\left(\gamma_{2}\right), \ldots, f\left(\gamma_{N}\right)\right) \in \mathbb{R}^{N} .
$$

With this identification, it is easy to see that the subset of $\mathbb{R}^{N}$ corresponding to $\left(\mathbb{R}^{+}\right)^{\Gamma, \succeq}$ is a rational convex polyhedral cone. Let $\mathbb{Z}^{\Gamma}$ be the space of all integer-valued functions on $\Gamma$ (which can be identified with the lattice $\mathbb{Z}^{N}$ in $\mathbb{R}^{N}$ ) and let

$$
\left(\mathbb{Z}^{+}\right)^{\Gamma, \succeq}=\left(\mathbb{R}^{+}\right)^{\Gamma, \succeq} \cap \mathbb{Z}^{\Gamma} .
$$

Then $\left(\mathbb{Z}^{+}\right)^{\Gamma, \succeq}$ is a lattice cone. It provides a natural setting for the affine semigroup rings studied by Hibi in [Hi]. Therefore, we will call it a Hibi cone. See also [Ho3, Ki1].

We now describe the semigroup structure of $\left(\mathbb{Z}^{+}\right)^{\Gamma, \succeq}$. A subset $A$ of $\Gamma$ is said to be increasing if for any $a \in A$,

$$
x \in \Gamma \text { and } x \succeq a \Longrightarrow x \in A .
$$

Let $J^{*}(\Gamma)$ be the collection of increasing subsets of $\Gamma$. For each $A \in J^{*}(\Gamma)$, let $\chi_{A}: \Gamma \rightarrow \mathbb{Z}^{+}$ be the characteristic function of $A$, that is,

$$
\chi_{A}(x)= \begin{cases}1 & x \in A \\ 0 & x \notin A\end{cases}
$$

(If $A=\emptyset$, then $\chi_{A}(x)=0$ for all $x \in \Gamma$.)

Theorem 3.4. ([Ho3]) The semigroup $\left(\mathbb{Z}^{+}\right)^{\Gamma, \succeq}$ is generated by $\left\{\chi_{A}: A \in J^{*}(\Gamma)\right\}$. More precisely, each element $f$ of $\left(\mathbb{Z}^{+}\right)^{\Gamma, \succeq}$ has a unique expression as a sum

$$
f=\sum_{j=1}^{u} c_{j} \chi_{A_{j}}
$$

where $c_{j}$ are positive integers and $\emptyset \subsetneq A_{1} \subsetneq A_{2} \subsetneq \cdots \subsetneq A_{u}$ is a chain in the poset $J^{*}(\Gamma)$.

3.6. Standard monomial theory. Let $R$ be a complex algebra and let $\mathcal{G}$ be a finite set of elements of $R$ with a partial ordering. If $g_{1} \leq g_{2} \leq \cdots \leq g_{s}$ in $\mathcal{G}$ (i.e. the $g_{i}$ form a multichain in $\mathcal{G}$ ), then we call the product

$$
g_{1} g_{2} \cdots g_{s}
$$

a standard monomial on $\mathcal{G}$. Let $\mathcal{B}$ be the set of all standard monomials on $\mathcal{G}$. If $\mathcal{B}$ forms a basis for $R$, then we call $\mathcal{B}$ a standard monomial basis and say that $R$ has a standard monomial theory with respect to $\mathcal{G}$. 
3.7. The structure of $\mathcal{P}\left(\mathrm{M}_{n, m}\right)^{U_{n} \times U_{k}}$. We resume our description of the structure of the algebra $\mathcal{P}\left(\mathrm{M}_{n, m}\right)^{U_{n} \times U_{k}}$. Recall that $m=k+\ell$. Fix Young diagrams $D$ and $F$ with $r(D) \leq \min (n, k)$ and $r(F) \leq \min (n, m)$, and $\alpha \in\left(\mathbb{Z}^{+}\right)^{\ell}$. We shall specify a basis for the homogeneous component $W_{F, D, \alpha}$ of $\mathcal{P}\left(\mathrm{M}_{n, m}\right)^{U_{n} \times U_{k}}$.

A column skew tableau is defined by a certain number $p$ of empty boxes at the top, followed by boxes labeled by a strictly increasing sequence $S=\left\{s_{1}, s_{2}, \ldots, s_{q}\right\}$ of positive integers. Let us label this column skew tableau $T_{(p, S)}$. For every column tableau $T_{(p, S)}$ with $p \leq \min (n, k), p+q \leq \min (n, m)$ and $s_{q} \leq \ell$, we define an element of the algebra $\mathcal{P}\left(\mathrm{M}_{n, m}\right)^{U_{n} \times U_{k}}$ by the determinant

$$
\delta_{(p, S)}=\left|\begin{array}{cccccccc}
x_{11} & x_{12} & \cdots & x_{1 p} & y_{1 s_{1}} & y_{1 s_{2}} & \cdots & y_{1 s_{q}} \\
x_{21} & x_{22} & \cdots & x_{2 p} & y_{2 s_{1}} & y_{2 s_{2}} & \cdots & y_{2 s_{q}} \\
\vdots & \vdots & & \vdots & \vdots & \vdots & & \vdots \\
x_{(p+q) 1} & x_{(p+q) 2} & \cdots & x_{(p+q) p} & y_{(p+q) s_{1}} & y_{(p+q) s_{2}} & \cdots & y_{(p+q) s_{q}}
\end{array}\right| .
$$

Now consider any semistandard skew tableau $T$ in $\mathrm{ST}_{(F, D, \alpha)}$. We will define a polynomial $\delta_{T}$ in $W_{F, D, \alpha}$ and a monomial $m_{T}$ as follows. Let the $j$-th column (counting from left to right) of $T$ be $T_{j}=T_{\left(p_{j}, S_{j}\right)}$. Then $\delta_{T}$ is defined as

$$
\delta_{T}=\prod_{j=1}^{c} \delta_{T_{j}}=\prod_{j=1}^{c} \delta_{\left(p_{j}, S_{j}\right)},
$$

where $c$ is the number of columns in $T$. To define the monomial $m_{T}$, let $\left(F_{0}, F_{1}, \ldots, F_{\ell}\right)$ be the sequence of Young diagrams which corresponds to $T$ under the bijection defined in equation (3.14). Since $r\left(F_{0}\right)=r(D) \leq \min (n, k)$ and $D=F_{0} \sqsubseteq F_{1} \sqsubseteq \cdots \sqsubseteq F_{\ell-1} \sqsubseteq F_{\ell}=F$, $r\left(F_{j}\right) \leq \min (n, k+j)$ for $0 \leq j \leq \ell$. So we can write for each $0 \leq j \leq \ell$,

$$
F_{j}=\left(\lambda_{1 j}, \lambda_{2 j}, \ldots, \lambda_{\min (n, k+j) j}\right) .
$$

Then

$$
m_{T}=\left(\prod_{i} x_{i i}^{\lambda_{i 0}}\right)\left(\prod_{a, b} y_{a b}^{\lambda_{a b}-\lambda_{a(b-1)}}\right) .
$$

The polynomial $\delta_{T}$ and the monomial $m_{T}$ are related as follows. Let the set of monomials in $\mathcal{P}\left(\mathrm{M}_{n, m}\right)$ be given the graded lexicographic order with respect to the following ordering on the variables:

(a) $x_{a b}>x_{c d}$ iff $b<d$ or $b=d$ and $a<c$.

(b) Similarly, $y_{a b}>y_{c d}$ iff $b<d$ or $b=d$ and $a<c$.

(c) Finally, $x_{a b}>y_{c d}$ for all pairs $(a, b)$ and $(c, d)$ of indices.

Then it can be checked that (see [HL2, Ki4]) for each semistandard tableau $T \in \mathrm{ST}_{(F, D, \alpha)}$,

$$
\operatorname{LM}\left(\delta_{T}\right)=m_{T} .
$$

Theorem 3.5. (i) The set $\mathcal{B}_{F, D, \alpha}=\left\{\delta_{T}: T \in \mathrm{ST}_{(F, D, \alpha)}\right\}$ is a basis for $W_{F, D, \alpha}$.

(ii) The set $\mathcal{B}=\left\{\delta_{T}: T \in \mathrm{ST}_{(n, k, \ell)}\right\}$ is a basis for $\mathcal{P}\left(\mathrm{M}_{n, m}\right)^{U_{n} \times U_{k}}$.

(iii) $\operatorname{LM}\left(\mathcal{P}\left(\mathrm{M}_{n, m}\right)^{U_{n} \times U_{k}}\right)=\left\{m_{T}: T \in \mathrm{ST}_{(n, k, \ell)}\right\}$. 


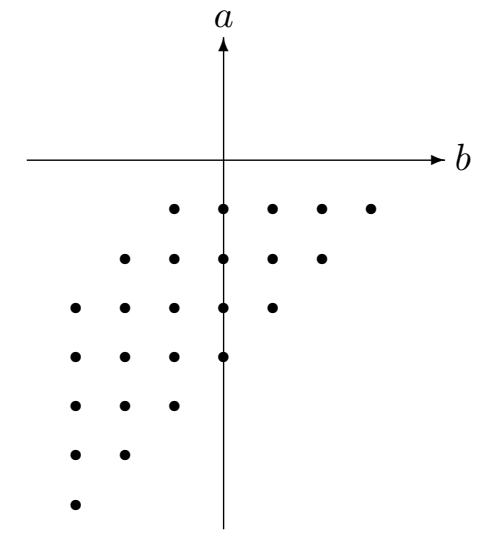

Figure 1. The set $\Gamma_{(7,3,4)}$.

Proof. By equation (3.21), the leading monomials $\operatorname{LM}\left(\delta_{T}\right)$ are distinct. So the set $\mathcal{B}_{F, D, \alpha}$ is linearly independent. Moreover, the number of vectors in $\mathcal{B}_{F, D, \alpha}$ coincides with $\operatorname{dim} W_{F, D, \alpha}$. So (i) follows. Part (ii) follows from (i) and the decomposition (3.10). For (iii), we first note that $m_{T}=\operatorname{LM}\left(\delta_{T}\right) \in \operatorname{LM}\left(\mathcal{P}\left(\mathrm{M}_{n, m}\right)^{U_{n} \times U_{k}}\right)$ for each $T \in \mathrm{ST}_{(n, k, \ell)}$. Next, we let $p \in \mathcal{P}\left(\mathrm{M}_{n, m}\right)^{U_{n} \times U_{k}}$. By (ii),

$$
p=c_{1} \delta_{T_{1}}+c_{2} \delta_{T_{2}}+\cdots+c_{r} \delta_{T_{r}}
$$

for some $c_{1}, \ldots, c_{r} \in \mathbb{C}^{\times}$and $T_{1}, \ldots, T_{r} \in \mathrm{ST}_{(n, k, \ell)}$. Since the leading monomials $\operatorname{LM}\left(\delta_{T_{1}}\right)=$ $m_{T_{1}}, \ldots, \operatorname{LM}\left(\delta_{T_{r}}\right)=m_{T_{r}}$ are distinct, we have $\operatorname{LM}(p)=m_{T_{j}}$ for some $1 \leq j \leq r$.

3.8. The Hibi cone associated with $\mathcal{P}\left(\mathrm{M}_{n, m}\right)^{U_{n} \times U_{k}}$. In this subsection, we shall show that the semigroup $\operatorname{LM}\left(\mathcal{P}\left(\mathrm{M}_{n, m}\right)^{U_{n} \times U_{k}}\right)$ is a Hibi cone. This cone is closely related to the Gelfand-Tsetlin cone, and we could describe it in terms of truncated Gelfand-Tsetlin patterns. However, in preparation for Section 4, it is convenient to embed the associated poset in $\mathbb{Z}^{2}$, and this is what we will do here.

For $0 \leq j \leq \ell$ and $1 \leq i \leq \min (k+j, n)$, let

$$
\gamma_{i}^{(j)}=\left[\begin{array}{c}
j-i \\
-i
\end{array}\right] \in \mathbb{Z}^{2}
$$

Consider the set of points

$$
\Gamma_{(n, k, \ell)}=\left\{\gamma_{i}^{(j)}: 0 \leq j \leq \ell, 1 \leq i \leq \min (k+j, n)\right\}
$$

in the plane. The set $\Gamma_{(7,3,4)}$ is illustrated in Figure 1 .

We consider $\mathbb{Z}^{2}$ as a poset with respect to the usual partial order

$$
\left[\begin{array}{l}
a \\
b
\end{array}\right] \preceq\left[\begin{array}{c}
c \\
d
\end{array}\right] \text { if and only if } a \leq c \text { and } b \leq d,
$$

and we consider $\Gamma_{(n, k, \ell)}$ as a poset with the induced partial ordering. Consider the lattice cone $\left(\mathbb{Z}^{+}\right)^{\Gamma_{(n, k, \ell)}, \succeq}$ associated with $\Gamma_{(n, k, \ell)}$ (see equation (3.18)). 
Lemma 3.6. There is a bijection $\Psi: \mathrm{ST}_{(n, k, \ell)} \rightarrow\left(\mathbb{Z}^{+}\right)^{\Gamma_{(n, k, \ell)}, \succeq}$.

Proof. Given a semistandard tableau $T$ in $\mathrm{ST}_{(n, k, \ell)}$, we define a function $f_{T}$ on $\Gamma_{(n, k, \ell)}$ by

$$
f_{T}\left(\gamma_{i}^{(j)}\right)=\lambda_{i j}
$$

where $\lambda_{i j}$ is defined in equation (3.20). It is easy to check that $f_{T}$ is an increasing function on the poset $\Gamma_{(n, k, \ell)}$, and conversely, that every increasing function $f: \Gamma_{(n, k, \ell)} \rightarrow \mathbb{Z}^{+}$is of the form $f=f_{T}$ for some $T \in \mathrm{ST}_{(n, k, \ell)}$. It follows that the map

$$
\Psi(T)=f_{T}, \quad T \in \mathrm{ST}_{(n, k, \ell)},
$$

is a bijection from $\mathrm{ST}_{(n, k, \ell)}$ to $\left(\mathbb{Z}^{+}\right)^{\Gamma_{(n, k, \ell)}, \succeq}$.

Corollary 3.7. The map

$$
\begin{aligned}
\operatorname{LM}\left(\mathcal{P}\left(\mathrm{M}_{n, m}\right)^{U_{n} \times U_{k}}\right) & \longrightarrow\left(\mathbb{Z}^{+}\right)^{\Gamma_{(n, k, \ell)}, \succeq} \\
m_{T} & \longmapsto f_{T}
\end{aligned}
$$

is a semigroup isomorphism.

By Theorem 3.4, $\left(\mathbb{Z}^{+}\right)^{\Gamma_{(n, k, \ell)}, \succeq}$ is generated by $\left\{\chi_{A}: A \in J^{*}\left(\Gamma_{(n, k, \ell)}\right)\right\}$. In particular, it is finitely generated. It follows from this and Theorem 3.3 that

Theorem 3.8. There exists a flat one-parameter family of $\mathbb{C}$-algebras with general fibre $\mathcal{P}\left(\mathrm{M}_{n, m}\right)^{U_{n} \times U_{k}}$ and special fibre $\mathbb{C}\left[\left(\mathbb{Z}^{+}\right)^{\Gamma(n, k, \ell)}, \succeq\right]$.

Next, we shall describe the increasing subsets of the poset $\Gamma_{(n, k, \ell)}$. For a nonnegative integer $p$ and a set $S \subseteq\{1,2, \ldots, \ell\}$ with $p \leq \min (n, k)$ and $|S| \leq \min (n, m)-p$, we define the sequence $\left(a_{0}, a_{1}, \ldots, a_{\ell}\right)$ as follows:

(i) $a_{0}=p$.

(ii) For $1 \leq t \leq \ell-1$,

$$
a_{t+1}= \begin{cases}a_{t}+1 & t+1 \in S \\ a_{t} & t+1 \notin S\end{cases}
$$

We now let

$$
A_{(p, S)}=\left\{\gamma_{i}^{(j)} ; 1 \leq i \leq a_{i}, 0 \leq j \leq \ell\right\} .
$$

(So $A_{(0, \emptyset)}=\emptyset$.) It is easy to show that $A_{(p, S)}$ is increasing. In fact, these sets exhaust all the increasing subsets of $\Gamma_{(n, k, \ell)}$.

Lemma 3.9. We have

$$
J^{*}\left(\Gamma_{n, k, \ell}\right)=\left\{A_{(p, S)}: 0 \leq p \leq \min (n, k), S \subseteq\{1,2, \ldots, \ell\},|S| \leq \min (n, m)-p\right\} .
$$

Proof. Let $B$ be an increasing subset of $\Gamma_{(n, k, \ell)}$. Then

$$
B=\left\{\gamma_{i}^{(j)}: 1 \leq i \leq a_{i}, 0 \leq j \leq \ell\right\}
$$

for some $a_{0}, \ldots, a_{\ell} \geq 0$.

Let $0 \leq t \leq \ell-1$. Then $\gamma_{a_{t}}^{(t)} \in B$ and $\gamma_{a_{t}+1}^{(t)} \notin B$. Since $B$ is increasing and $\gamma_{a_{t}}^{(t+1)} \geq \gamma_{a_{t}}^{(t)}$, $\gamma_{a_{t}}^{(t+1)} \in B$. Further, since $\gamma_{a_{t}+2}^{(t+1)} \leq \gamma_{a_{t}+1}^{(t)}$ and $\gamma_{a_{t}+1}^{(t)} \notin B, \gamma_{a_{t}+2}^{(t+1)} \notin B$. So $a_{t+1}=a_{t}$ or $a_{t}+1$. We now let $p=a_{0}$ and define the set $S$ by equations (3.26). Then $B=A_{(p, S)}$. 
It is not difficult to check that under the bijection $\Psi$ defined in Lemma 3.6, the column tableau $T_{(p, S)}$ is mapped to the function $\chi_{A(p, S)}$. We now let $\mathcal{G}_{(n, k, \ell)}$ be the set of all polynomials of the form $\delta_{(p, S)}$, and define a partial order on $\mathcal{G}_{n, k, \ell}$ by

$$
\delta_{(p, S)} \geq \delta_{\left(p^{\prime}, S^{\prime}\right)} \Longleftrightarrow A_{(p, S)} \supseteq A_{\left(p^{\prime}, S^{\prime}\right)} .
$$

This partial order is the opposite of the standard partial order on the set of column tableaux ([HL2, Ki4]). Let $T \in \mathrm{ST}_{n, k, \ell}$. Suppose that it has $c$ columns and $T_{j}=T_{\left(p_{j}, S_{j}\right)}$ is its $j$-th column. Consider the polynomial $\delta_{T}$ defined in equation (3.19) . Then we observe that

$$
A_{\left(p_{1}, S_{1}\right)} \supseteq A_{\left(p_{2}, S_{2}\right)} \supseteq \cdots \supseteq A_{\left(p_{c}, S_{c}\right)} .
$$

This means that $\delta_{T}$ is a standard monomial on the elements in $\mathcal{G}_{(n, k, \ell)}$ with respect to the above partial order. Hence the basis $\mathcal{B}$ of $\mathcal{P}\left(\mathrm{M}_{n, m}\right)^{U_{n} \times U_{k}}$ defined in Theorem 3.5 is a standard monomial basis.

Remark. We would like to highlight the extent to which the logic of this account depends on the leading monomials. First, using the skew tableau technology, one finds a combinatorial parametrization of the constituents of the tensor products that counts the multiplicity of any given representation in the indicated tensor products. Then one encodes the parameters as a collection of exponents, and observes that these exponents exactly constitute a lattice cone, isomorphic to the Hibi cone $\left(\mathbb{Z}^{+}\right)^{\Gamma_{(n, k, \ell)}, \succeq}$.

Finally, one defines a monomial order and produces highest weight vectors for $\mathrm{GL}_{n}$ whose leading monomials have the previously described exponents. These highest weight vectors are monomials in an explicit set $\mathcal{G}_{(n, k, \ell)}$ of elements. The fact that these monomials have distinct highest terms guarantees that they are linearly independent, and therefore, the monomials for a given highest weight span a space of the required dimension. These monomials therefore exhaust the highest weight vectors of a given type, and therefore span the algebra being studied. It is the upper bound given by the combinatorial parameters, together with the lower bound given by the distinctness of the highest terms, and the matching of leading monomials with combinatorial parameters, that guarantees everything is found.

In this reasoning, we have paid no direct attention to the algebra structure of $\mathcal{P}\left(\mathrm{M}_{n, m}\right)^{U_{n} \times U_{k}}$. We find at the end that the $\mathcal{G}_{(n, k, \ell)}$ must be generators for the algebra, but we have not given any consideration to the relations between them. Thanks to general SAGBI theory, we have exhibited $\mathcal{P}\left(\mathrm{M}_{n, m}\right)^{U_{n} \times U_{k}}$ as a flat deformation of the Hibi ring on the poset $\Gamma_{(n, k, \ell)}$, and we note that the relations for a Hibi ring are easily described (see Theorem 3.4). Although this does not determine the relations between the elements in $\mathcal{G}_{(n, k, \ell)}$, it does allow one to make some conclusions about their qualitative nature. Detailed exploration of this topic is beyond the scope of this article. See [Ki4].

\section{The double Pieri algebras}

In this section, we construct a family $\mathcal{L}_{(n, p),(k, q)}$ of algebras and study their structure. The process for constructing it suggests the name double Pieri algebra. Later we will show that this algebra is closely related to the iterated Pieri algebras for $\mathrm{GL}_{n}$, and also for $\mathrm{O}_{n}$ and $\mathrm{Sp}_{2 n}$. More precisely, we shall show that under certain conditions on the parameters, each of the iterated Pieri algebras is essentially made up by either one or two algebras of the form $\mathcal{L}_{(n, p),(k, q)}$ together with a polynomial algebra. Thus, the iterated Pieri algebras for all three series of classical groups are unified via the double Pieri algebra. 
4.1. The construction of $\mathcal{L}_{(n, p),(k, q)}$. In Section 3.3, we were concerned with the $\mathrm{GL}_{n}$ structure of the polynomial iterated Pieri algebra

$$
\mathcal{P}\left(\mathrm{M}_{n, k+p}\right)^{U_{n} \times U_{k}} \simeq\{\mathcal{P}\left(\mathrm{M}_{n, k}\right) \otimes \overbrace{\mathcal{P}\left(\mathbb{C}^{n}\right) \otimes \cdots \otimes \mathcal{P}\left(\mathbb{C}^{n}\right)}^{p}\}^{U_{n} \times U_{k}} .
$$

However, the starting situation of $\mathcal{P}\left(\mathrm{M}_{n, k}\right)$ is symmetric in $n$ and $k$, and we can clearly tensor $\mathcal{P}\left(\mathrm{M}_{n, k}\right)$ with copies of $\mathcal{P}\left(\mathbb{C}^{k}\right)$ and get an analogous decomposition of the resulting $\mathrm{GL}_{k}$ module.

In fact, we can do both at the same time. Let $n, k, p, q$ be integers such that $n \geq k \geq 1$ and $p, q \geq 0$, and consider the algebra $\mathcal{P}\left(\mathrm{M}_{n, k} \oplus \mathrm{M}_{n, p} \oplus \mathrm{M}_{q, k}\right)$ of polynomial functions on $\mathrm{M}_{n, k} \oplus \mathrm{M}_{n, p} \oplus \mathrm{M}_{q, k}$. We let $\mathrm{GL}_{n} \times \mathrm{GL}_{k} \times \mathrm{GL}_{p} \times \mathrm{GL}_{q}$ act on $\mathcal{P}\left(\mathrm{M}_{n, k} \oplus \mathrm{M}_{n, p} \oplus \mathrm{M}_{q, k}\right)$ by the formula:

$$
(\sigma(g, h, a, b)(f))(X, Y, R)=f\left(g^{t} X h, g^{t} Y a, b^{t} R h\right)
$$

where $(g, h, a, b) \in \mathrm{GL}_{n} \times \mathrm{GL}_{k} \times \mathrm{GL}_{p} \times \mathrm{GL}_{q}, f \in \mathcal{P}\left(\mathrm{M}_{n, k} \oplus \mathrm{M}_{n, p} \oplus \mathrm{M}_{q, k}\right)$ and $(X, Y, R) \in$ $\mathrm{M}_{n, k} \oplus \mathrm{M}_{n, p} \oplus \mathrm{M}_{q, k}$. Also, we restrict the action to the subgroup $\mathrm{GL}_{n} \times \mathrm{GL}_{k} \times A_{p} \times A_{q}$. Finally, we let

$$
\mathcal{L}_{(n, p),(k, q)}=\left[\mathcal{P}\left(\mathrm{M}_{n, k} \oplus \mathrm{M}_{n, p} \oplus \mathrm{M}_{q, k}\right)\right]^{U_{n} \times U_{k}}
$$

be the subalgebra of $U_{n} \times U_{k}$ invariants in $\mathcal{P}\left(\mathrm{M}_{n, k} \oplus \mathrm{M}_{n, p} \oplus \mathrm{M}_{q, k}\right)$ and call it a double Pieri algebra. It is a module for $A_{n} \times A_{p} \times A_{k} \times A_{q}$. It turns out that we can analyze it by an extension of ideas on the polynomial iterated Pieri algebra $\mathcal{P}\left(\mathrm{M}_{n, k+p}\right)^{U_{n} \times U_{k}}$.

4.2. Multigraded structure of $\mathcal{L}_{(n, p),(k, q)}$. The torus $A_{n} \times A_{p} \times A_{k} \times A_{q}$ acts on $\mathcal{L}_{(n, p),(k, q)}$ by algebra automorphisms, so $\mathcal{L}_{(n, p),(k, q)}$ is a multi-graded algebra. More precisely, we have

$$
\mathcal{L}_{(n, p),(k, q)}=\sum_{F, \alpha, D, \beta} W_{(F, \alpha),(D, \beta)}
$$

where the sum is taken over all Young diagrams $F$ and $D$ with $r(F) \leq \min (n, k+p)$ and $r(D) \leq k, \alpha \in\left(\mathbb{Z}^{+}\right)^{p}$ and $\beta \in\left(\mathbb{Z}^{+}\right)^{q}$, and the homogeneous component $W_{(F, \alpha),(D, \beta)}$ is the $\psi_{n}^{F} \times \psi_{p}^{\alpha} \times \psi_{k}^{D} \times \psi_{q}^{\beta}$ eigenspace of $A_{n} \times A_{p} \times A_{k} \times A_{q}$ in $\mathcal{L}_{(n, p),(k, q)}$.

\section{Notation.}

(i) For Young diagrams $F$ and $D$, and $\alpha \in\left(\mathbb{Z}^{+}\right)^{p}$ and $\beta \in\left(\mathbb{Z}^{+}\right)^{q}$, let $\mathrm{ST}_{(F, \alpha),(D, \beta)}$ be the set of ordered pairs $\left(T_{1}, T_{2}\right)$ of semistandard tableaux satisfying the following conditions: There is a Young diagram $E$ such that $T_{1}$ is of shape $F / E$ and content $\alpha$, and $T_{2}$ is of shape $D / E$ and content $\beta$. (So $T_{1}$ and $T_{2}$ have the same initial Young diagram $E$.)

(ii) Let $K_{(F, \alpha),(D, \beta)}$ denote the number of elements in the set $\mathrm{ST}_{(F, \alpha),(D, \beta)}$. Then

$$
K_{(F, \alpha),(D, \beta)}=\sum_{E} K_{F / E, \alpha} K_{D / E, \beta} .
$$

(iii) We also let

$$
\mathrm{ST}_{(n, p),(k, q)}=\bigcup_{(F, \alpha),(D, \beta)} \mathrm{ST}_{(F, \alpha),(D, \beta)}
$$

where the union is taken over all pairs $(F, \alpha)$ and $(D, \beta)$ with $r(F) \leq \min (n, k+p)$, $\alpha \in\left(\mathbb{Z}^{+}\right)^{p}, r(D) \leq k$ and $\beta \in\left(\mathbb{Z}^{+}\right)^{q}$. 
Lemma 4.1. The dimension of $W_{(F, \alpha)(D, \beta)}$ is given by

$$
\operatorname{dim} W_{(F, \alpha)(D, \beta)}=K_{(F, \alpha),(D, \beta)} .
$$

Proof. Let $\mathrm{GL}_{n} \times \mathrm{GL}_{k} \times A_{p} \times A_{q}$ act on $\mathcal{P}\left(\mathrm{M}_{n, k} \oplus \mathrm{M}_{n, p} \oplus \mathrm{M}_{q, k}\right)$ via the action $\sigma$ given in equation (4.1). From (3.8), by setting $k=0$ and $\ell=p$, we have

$$
\mathcal{P}\left(\mathrm{M}_{n, p}\right) \simeq \sum_{\alpha=\left(\alpha_{i}\right) \in\left(\mathbb{Z}^{+}\right)^{p}}\left(\bigotimes_{i=1}^{p} \rho_{n}^{\left(\alpha_{i}\right)}\right) \otimes \psi_{p}^{\alpha}
$$

Similarly, we also have

$$
\mathcal{P}\left(\mathrm{M}_{q, k}\right) \simeq \sum_{\beta=\left(\beta_{i}\right) \in\left(\mathbb{Z}^{+}\right)^{q}}\left(\psi_{q}^{\beta} \otimes \bigotimes_{i=1}^{q} \rho_{k}^{\left(\beta_{i}\right)}\right)
$$

Then, by $\left(\mathrm{GL}_{n}, \mathrm{GL}_{k}\right)$-duality and the polynomial iterated Pieri rule for $\mathrm{GL}_{n}$ given in equation (3.15), we have

$$
\begin{aligned}
& \mathcal{P}\left(\mathrm{M}_{n, k} \oplus \mathrm{M}_{n, p} \oplus \mathrm{M}_{q, k}\right) \simeq \mathcal{P}\left(\mathrm{M}_{n, k}\right) \otimes \mathcal{P}\left(\mathrm{M}_{n, p}\right) \otimes \mathcal{P}\left(\mathrm{M}_{q, k}\right) \\
\simeq & \left\{\sum_{r(E) \leq k} \rho_{n}^{E} \otimes \rho_{k}^{E}\right\} \otimes\left\{\sum_{\alpha=\left(\alpha_{i}\right) \in\left(\mathbb{Z}^{+}\right)^{p}}\left(\bigotimes_{i=1}^{p} \rho_{n}^{\left(\alpha_{i}\right)}\right) \otimes \psi_{p}^{\alpha}\right\} \otimes\left\{\sum_{\beta=\left(\beta_{i}\right) \in\left(\mathbb{Z}^{+}\right)^{q}}\left(\psi_{q}^{\beta} \otimes \bigotimes_{i=1}^{q} \rho_{k}^{\left(\beta_{i}\right)}\right)\right\} \\
\simeq & \sum_{E, \alpha, \beta}\left(\rho_{n}^{E} \otimes\left(\bigotimes_{i=1}^{p} \rho_{n}^{\left(\alpha_{i}\right)}\right)\right) \otimes\left(\rho_{k}^{E} \otimes\left(\bigotimes_{i=1}^{q} \rho_{k}^{\left(\beta_{i}\right)}\right)\right) \otimes \psi_{p}^{\alpha} \otimes \psi_{q}^{\beta} \\
\simeq & \sum_{E, \alpha, \beta}\left(\sum_{F} K_{F / E, \alpha} \rho_{n}^{F}\right) \otimes\left(\sum_{D} K_{D / E, \beta} \rho_{k}^{D}\right) \otimes \psi_{p}^{\alpha} \otimes \psi_{q}^{\beta} \\
\simeq & \sum_{\alpha, \beta, D, F}\left(\sum_{E} K_{F / E, \alpha} K_{D / E, \beta}\right) \rho_{n}^{F} \otimes \rho_{k}^{D} \otimes \psi_{p}^{\alpha} \otimes \psi_{q}^{\beta} \\
\simeq & \sum_{\alpha, \beta, D, F} K_{(F, \alpha),(D, \beta)} \rho_{n}^{F} \otimes \rho_{k}^{D} \otimes \psi_{p}^{\alpha} \otimes \psi_{q}^{\beta},
\end{aligned}
$$

where all the Young diagrams $F$ and $D$ which appear in the sum are such that $r(F) \leq$ $\min (n, k+p)$ and $r(D) \leq k$. The lemma follows by extracting $U_{n} \times U_{k}$ invariants from these expressions.

4.3. Generators of $\mathcal{L}_{(n, p),(k, q)}$. We denote the standard coordinates on $\mathrm{M}_{n, k}, \mathrm{M}_{n, p}$ and $\mathrm{M}_{q, k}$ as

$$
\left(x_{i j}\right) \in \mathrm{M}_{n, k}, \quad\left(y_{i j}\right) \in \mathrm{M}_{n, p}, \quad\left(z_{i j}\right) \in \mathrm{M}_{q, k},
$$

so that $\mathcal{P}\left(\mathrm{M}_{n, k} \oplus \mathrm{M}_{n, p} \oplus \mathrm{M}_{q, k}\right)$ can be regarded as a polynomial algebra on these variables. Let the set of monomials in $\mathcal{P}\left(\mathrm{M}_{n, k} \oplus \mathrm{M}_{n, p} \oplus \mathrm{M}_{q, k}\right)$ be given the graded lexicographic order with respect to following ordering on the variables:

(a) $x_{a b}>x_{c d}$ iff $b<d$ or $b=d$ and $a<c$.

(b) $y_{a b}>y_{c d}$ iff $b<d$ or $b=d$ and $a<c$.

(c) $z_{a b}>z_{c d}$ iff $a<c$ or $a=c$ and $b<d$.

(d) Finally, $x_{a b}>y_{c d}>z_{e f}$ for all pairs $(a, b),(c, d)$ and $(e, f)$ of indices. 
We want to construct a basis for the double Pieri algebra $\mathcal{L}_{(n, p),(k, q)}$. Lemma 4.1 suggests that we can do so by attaching an element $\delta_{\left(T, T^{\prime}\right)}$ of $\mathcal{L}_{(n, p),(k, q)}$ to each pair $\left(T, T^{\prime}\right)$ of semistandard tableaux in $\mathrm{ST}_{(n, p),(k, q)}$. The most basic case is when both $T$ and $T^{\prime}$ are column tableaux, that is, $T=T_{(c, J)}$ and $T^{\prime}=T_{(c, I)}$ where $0 \leq c \leq k$, and

$$
\begin{aligned}
I & =\left\{i_{1}<i_{2}<\cdots<i_{u}\right\} \subseteq\{1, \ldots, q\}, \\
J & =\left\{j_{1}<j_{2}<\cdots<j_{v}\right\} \subseteq\{1, \ldots, p\}
\end{aligned}
$$

with $u+c \leq k$ and $v+c \leq \min (n, k+p)$. Let $\delta_{(c, I, J)}$ be the determinant

$$
\delta_{(c, I, J)}=\left|\begin{array}{ccccccc}
x_{1,1} & x_{1,2} & \cdots & x_{1, c+u} & y_{1, j_{1}} & \cdots & y_{1, j_{v}} \\
x_{2,1} & x_{2,2} & \cdots & x_{2, c+u} & y_{2, j_{1}} & \cdots & y_{2, j_{v}} \\
\vdots & \vdots & & \vdots & \vdots & & \vdots \\
x_{c+v, 1} & x_{c+v, 2} & \cdots & x_{c+v, c+u} & y_{c+v, j_{1}} & \cdots & y_{c+v, j_{v}} \\
z_{i_{1}, 1} & z_{i_{1}, 2} & \cdots & z_{i_{1}, c+u} & 0 & \cdots & 0 \\
\vdots & \vdots & & \vdots & \vdots & & \vdots \\
z_{i_{u}, 1} & z_{i_{u}, 2} & \cdots & z_{i_{u}, c+u} & 0 & \cdots & 0
\end{array}\right| .
$$

The following lemma suggests that $\delta_{(c, I, J)}$ should be attached to $\left(T_{(c, J)}, T_{(c, I)}\right)$.

Lemma 4.2. The leading monomial of $\delta_{(c, I, J)}$ is given by

$$
\operatorname{LM}\left(\delta_{(c, I, J)}\right)=\left(\prod_{a=1}^{c} x_{a, a}\right)\left(\prod_{a=1}^{v} y_{c+a, j_{a}}\right)\left(\prod_{a=1}^{u} z_{i_{a}, c+a}\right) .
$$

Proof. Every term of the determinant $\delta_{(c, I, J)}$ is a product of $(c+u+v)$ elements chosen from each row and each column. To identify the leading monomial $\operatorname{LM}\left(\delta_{(c, I, J)}\right)$ of $\delta_{(c, I, J)}$, it is enough to choose the largest entry of the matrix at each step in making a product. It is straightforward to see that the first $c$ choices should be $x_{1,1}, \ldots, x_{c, c}$. After erasing the first $c$ rows and the first $c$ columns, we obtain the $(u+v) \times(u+v)$ subdeterminant

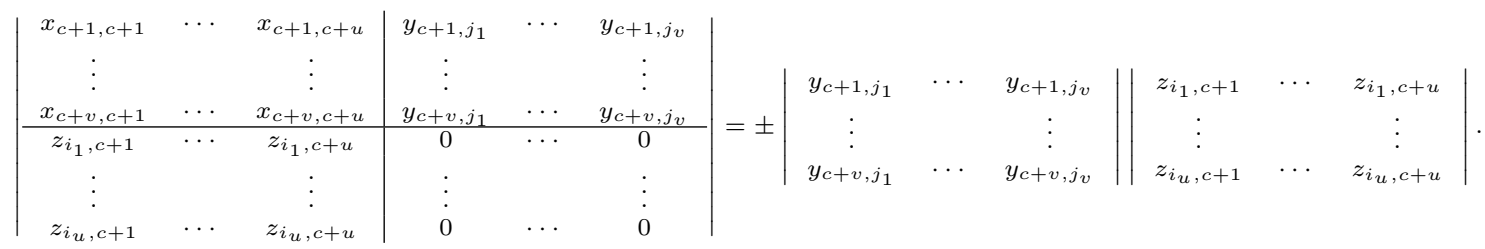

Clearly, the diagonal entries of the two determinants on the right hand side constitute the remaining entries in $\operatorname{LM}\left(\delta_{(c, I, J)}\right)$.

Later, we will define a partial order on the elements $\delta_{(c, I, J)}$ and show that standard monomials on these elements form a basis for $\mathcal{L}_{(n, p),(k, q)}$.

4.4. The Hibi cone associated to $\mathcal{L}_{(n, p),(k, q)}$. Let $\mathbb{Z}^{2}$ be given the partial ordering (3.24). Consider the reflection

$$
\tau:\left[\begin{array}{l}
a \\
b
\end{array}\right] \rightarrow\left[\begin{array}{l}
b \\
a
\end{array}\right]
$$

of $\mathbb{R}^{2}$. It clearly preserves $\mathbb{Z}^{2}$, and define an order preserving automorphism of $\mathbb{Z}^{2}$. Consider the sets of points $\Gamma_{(n, k, p)}$ and $\Gamma_{(k, n, q)}$ defined in equation (3.23), and let

$$
\Gamma_{(n, p),(k, q)}=\tau\left(\Gamma_{(k, n, q)}\right) \cup \Gamma_{(n, k, p)}
$$


where $\tau\left(\Gamma_{(k, n, q)}\right)$ is the image of $\Gamma_{(k, n, q)}$ under $\tau$. We consider $\Gamma_{(n, p),(k, q)}$ as a poset with the induced partial ordering. Explicitly, for integers $i$ and $a$, we let

$$
\gamma_{a}^{(i)}=\left\{\begin{array}{l}
{\left[\begin{array}{l}
-a \\
-a
\end{array}\right]+\left[\begin{array}{l}
i \\
0
\end{array}\right] \quad i \geq 0} \\
{\left[\begin{array}{l}
-a \\
-a
\end{array}\right]-\left[\begin{array}{l}
0 \\
i
\end{array}\right] \quad i<0 .}
\end{array}\right.
$$

Note that this definition extends that given in equation (3.22). Also, $\gamma_{a}^{(i)}=\tau\left(\gamma_{a}^{(-i)}\right)$ for $i<0$. Then

$$
\Gamma_{(n, p),(k, q)}=\left\{\gamma_{a}^{(i)}:-q \leq i \leq 0,1 \leq a \leq k\right\} \cup\left\{\gamma_{a}^{(i)}: 1 \leq i \leq p, 1 \leq a \leq \min (n, k+i)\right\}
$$

Two examples of $\Gamma_{(n, p),(k, q)}$ are illustrated in Figure 2 (with $n \geq k+p$ ) and Figure 3 (with $n<k+p$ ) respectively.

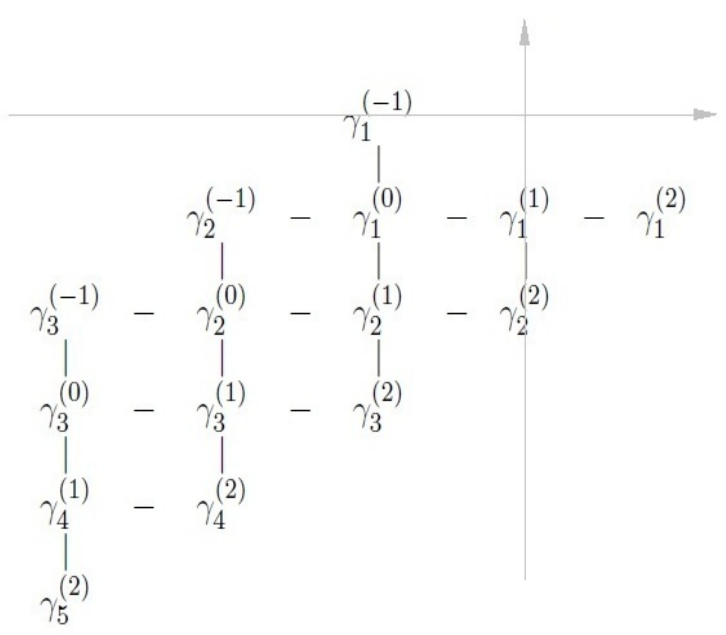

Figure 2. The set $\Gamma_{(5,2),(3,1)}$.

We now consider the Hibi cone $\left(\mathbb{Z}^{+}\right)^{\Gamma_{(n, p),(k, q)}, \succeq}$.

Lemma 4.3. There is a bijection $\Phi: \mathrm{ST}_{(n, p),(k, q)} \longrightarrow\left(\mathbb{Z}^{+}\right)^{\Gamma_{(n, p),(k, q)}, \succeq}$.

Proof. Let $\left(T, T^{\prime}\right) \in \mathrm{ST}_{(n, p),(k, q)}$. Consider the order preserving functions $f_{T}$ and $f_{T^{\prime}}$ defined in equation (3.25) on $\Gamma_{(n, k, p)}$ and $\Gamma_{(k, n, q)}$, respectively. For $u \in \tau\left(\Gamma_{(k, n, q)}\right)$, let

$$
f_{T^{\prime}}^{\tau}(u)=f_{T^{\prime}}(\tau(u))
$$

Then $f_{T^{\prime}}^{\tau}$ is an order preserving function on $\tau\left(\Gamma_{(k, n, q)}\right)$.

Now observe that

$$
\tau\left(\Gamma_{(k, n, q)}\right) \cap \Gamma_{(n, k, p)}=\left\{\gamma_{a}^{(0)}: 1 \leq a \leq k\right\} .
$$

Since $T$ and $T^{\prime}$ have the same initial diagram,

$$
f_{T}^{\tau}\left(\gamma_{a}^{(0)}\right)=f_{T^{\prime}}\left(\gamma_{a}^{(0)}\right)
$$




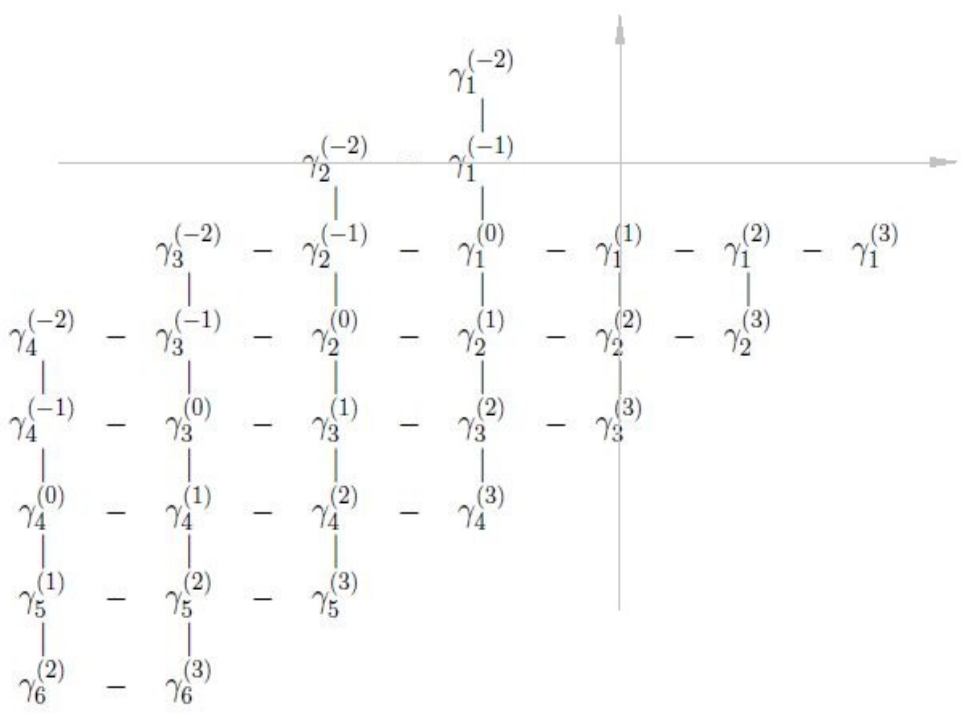

Figure 3. The set $\Gamma_{(6,3),(4,2)}$.

for each $1 \leq a \leq k$. This allows us to fit $f_{T}$ and $f_{T^{\prime}}^{\tau}$ together to define a function $f_{T, T^{\prime}}$ on $\Gamma_{(n, p),(k, q)}$ by

$$
f_{T, T^{\prime}}\left(\gamma_{j}^{(i)}\right)= \begin{cases}f_{T}\left(\gamma_{j}^{(i)}\right) & i \geq 0 \\ f_{T^{\prime}}^{\tau}\left(\gamma_{j}^{(i)}\right) & i \leq 0 .\end{cases}
$$

We claim that $f_{T, T^{\prime}}$ is order preserving. Suppose that $\gamma_{a}^{(i)} \in \Gamma_{(n, k, p)}, \gamma_{a}^{(-j)} \in \tau\left(\Gamma_{(k, n, q)}\right)$ and

$$
\gamma_{a}^{(i)}=\left[\begin{array}{c}
-a+i \\
-a
\end{array}\right] \succeq\left[\begin{array}{c}
-b \\
-b+j
\end{array}\right]=\gamma_{b}^{(-j)}
$$

(For example, $\gamma_{2}^{(3)} \succeq \gamma_{4}^{(-1)}$ in the poset $\Gamma_{(6,3),(4,2)}$. See Figure 3.) Then $-a \geq-b+j$, so that

$$
\gamma_{a}^{(i)}=\left[\begin{array}{c}
-a+i \\
-a
\end{array}\right] \succeq\left[\begin{array}{l}
-a \\
-a
\end{array}\right]=\gamma_{a}^{(0)} \succeq\left[\begin{array}{c}
-b \\
-b+j
\end{array}\right]=\gamma_{b}^{(-j)}
$$

It follows that

$$
f_{T, T^{\prime}}\left(\gamma_{a}^{(i)}\right)=f_{T}\left(\gamma_{a}^{(i)}\right) \geq f_{T}\left(\gamma_{a}^{(0)}\right)=f_{T^{\prime}}^{\tau}\left(\gamma_{a}^{(0)}\right) \geq f_{T^{\prime}}^{\tau}\left(\gamma_{b}^{(-j)}\right)=f_{T, T^{\prime}}\left(\gamma_{b}^{(-j)}\right) .
$$

Similarly, one can check that if $\gamma_{a}^{(i)} \preceq \gamma_{b}^{(-j)}$, then $f_{T, T^{\prime}}\left(\gamma_{a}^{(i)}\right) \leq f_{T, T^{\prime}}\left(\gamma_{b}^{(-j)}\right)$.

Conversely, each order preserving function $f$ on $\Gamma_{(n, p),(k, q)}$ is of the form $f=f_{T, T^{\prime}}$ for some $\left(T, T^{\prime}\right) \in \mathrm{ST}_{(n, p),(k, q)}$. This is easy to check. It follows that the map $\Phi: \mathrm{ST}_{(n, p),(k, q)} \rightarrow$

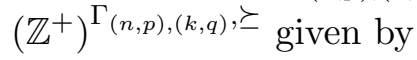

$$
\Phi\left(T, T^{\prime}\right)=f_{T, T^{\prime}}
$$

is a bijection. 
By Lemma 4.3 and equation (4.3),

$$
\left(\mathbb{Z}^{+}\right)^{\Gamma_{(n, p),(k, q)}, \succeq}=\bigcup_{(F, \alpha),(D, \beta)} \Phi\left(\mathrm{ST}_{(F, \alpha),(D, \beta)}\right) .
$$

If $f \in \Phi\left(\mathrm{ST}_{(F, \alpha),(D, \beta)}\right)$, then we can recover $(F, \alpha)$ and $(D, \beta)$ from $f$ as follows: Let us set

$$
f_{i}= \begin{cases}\left(f_{i, 1}, f_{i, 2}, \ldots, f_{i, k}\right) & \text { if }-q \leq i \leq 0 \\ \left(f_{i, 1}, f_{i, 2}, \ldots, f_{i, \min (n, k+i)}\right) & \text { if } 0<i \leq p\end{cases}
$$

where

$$
f_{i, j}=f\left(\gamma_{j}^{(i)}\right),
$$

and

$$
\begin{aligned}
& w^{+}(f)=\left(\left|f_{1}\right|-\left|f_{0}\right|,\left|f_{2}\right|-\left|f_{1}\right|, \ldots,\left|f_{p}\right|-\left|f_{p-1}\right|\right), \\
& w^{-}(f)=\left(\left|f_{-1}\right|-\left|f_{0}\right|,\left|f_{-2}\right|-\left|f_{-1}\right|, \ldots,\left|f_{-q}\right|-\left|f_{-q+1}\right|\right)
\end{aligned}
$$

where $\left|f_{i}\right|=\sum_{c \geq 1} f_{i, c}$ for $-q \leq i \leq p$. Then we have

$$
F=f_{p}, \quad \alpha=w^{+}(f), \quad D=f_{-q}, \quad \text { and } \beta=w^{-}(f) .
$$

Moreover, if $f=\Phi\left(T, T^{\prime}\right)$, then $f_{T}(u)=f(u)$ for all $u \in \Gamma_{n, k, \ell}$ and $f_{T^{\prime}}(v)=f(\tau(v))$ for all $v \in \Gamma_{k, n, q}$. This allows us to recover the tableaux $T$ and $T^{\prime}$.

It is also clear that if $f \in \Phi\left(\mathrm{ST}_{(F, \alpha),(D, \beta)}\right)$ and $f^{\prime} \in \Phi\left(\mathrm{ST}_{\left(F^{\prime}, \alpha^{\prime}\right),\left(D^{\prime}, \beta^{\prime}\right)}\right)$, then we have

$$
f+f^{\prime} \in \Phi\left(\mathrm{ST}_{\left(F+F^{\prime}, \alpha+\alpha^{\prime}\right),\left(D+D^{\prime}, \beta+\beta^{\prime}\right)}\right) \text {. }
$$

4.5. Increasing subsets of $\Gamma_{(n, p),(k, q)}$. By Theorem $3.4,\left(\mathbb{Z}^{+}\right)^{\Gamma_{(n, p),(k, q)}, \succeq}$ is generated by

$$
\left\{\chi_{A}: A \in J^{*}\left(\Gamma_{(n, p),(k, q)}\right)\right\} .
$$

We now specify the increasing subsets of $\Gamma_{(n, p),(k, q)}$. Our description is designed to make parallel with the special elements $\delta_{(c, I, J)}$ defined in equation (4.4). For $c, I=\left\{i_{1}, \ldots, i_{u}\right\} \subseteq$ $\{1, \ldots, q\}$, and $J=\left\{j_{1}, \ldots, j_{v}\right\} \subseteq\{1, \ldots, p\}$, where $0 \leq c \leq k,|I|=u \leq k-c$ and $|J|=v \leq$ $\min (n, k+p)-c$, we define a sequence $\left(a_{-q}, \ldots, a_{0}, \ldots, a_{p}\right)$ as follows:

(i) $a_{0}=c$,

(ii) For $0 \leq s \leq q-1$ and $0 \leq t \leq p-1$,

$$
a_{-s-1}= \begin{cases}a_{-s}+1 & s+1 \in\left\{i_{1}, \ldots, i_{u}\right\} \\ a_{-s} & s+1 \notin\left\{i_{1}, \ldots, i_{u}\right\}\end{cases}
$$

and

$$
a_{t+1}= \begin{cases}a_{t}+1 & t+1 \in\left\{j_{1}, \ldots, j_{v}\right\} \\ a_{t} & t+1 \notin\left\{j_{1}, \ldots, j_{v}\right\}\end{cases}
$$

Let

$$
A_{(c, I, J)}=\left\{\gamma_{j}^{(i)}: 1 \leq j \leq a_{i},-q \leq i \leq p\right\} .
$$

(So $A_{(0, \emptyset, \emptyset)}=\emptyset$.) Then one verifies that $A_{(c, I, J)}$ is increasing. In fact, the sets $A_{(c, I, J)}$ exhaust all the increasing subsets of $\Gamma_{(n, p),(k, q)}$.

Lemma 4.4. We have

$J^{*}\left(\Gamma_{(n, p),(k, q)}\right)=\left\{A_{(c, I, J)}: 0 \leq c \leq k, I \subseteq\{1, \ldots, q\}, J \subseteq\{1, \ldots, p\},|I| \leq k-c,|J| \leq \min (n, k+p)-c\right\}$. 
Proof. Let $B$ be an increasing subset of $\Gamma_{(n, p),(k, q)}$. Then $B=B_{1} \cup B_{2}$ where

$$
B_{1}=B \cap \tau\left(\Gamma_{k, n, q}\right) \text { and } B_{2}=B \cap \Gamma_{n, k, p} .
$$

Now $B_{1}$ is an increasing subset of $\tau\left(\Gamma_{k, n, q}\right)$, and since $\tau$ preserves the partial order of $\mathbb{Z}^{2}$, by Lemma $3.9, B_{1}=\tau\left(A_{(c, I)}\right)$ for some $0 \leq c \leq \min (k, n)$ and $I \subseteq\{1,2, \ldots, q\}$ with $|I| \leq k-c$. Similarly, $B_{2}=A_{(d, J)}$ for some $0 \leq d \leq \min (n, k)$ and $J \subseteq\{1,2, \ldots, p\}$ with $|J| \leq \min (n, k+p)-d$. Note that

$\left\{\gamma_{a}^{(0)}: 1 \leq a \leq c\right\}=B_{1} \cap \Gamma_{n, k, p}=B \cap \tau\left(\Gamma_{k, n, q}\right) \cap \Gamma_{n, k, p}=B_{2} \cap \tau\left(\Gamma_{k, n, q}\right)=\left\{\gamma_{a}^{(0)}: 1 \leq a \leq d\right\}$.

So $c=d$, and $B=A_{(c, I, J)}$.

4.6. Standard monomial basis for $\mathcal{L}_{(n, p),(k, q)}$. For each $f \in\left(\mathbb{Z}^{+}\right)^{\Gamma_{(n, p),(k, q)}, \succeq}$, we define the polynomial $\delta_{f} \in \mathcal{L}_{(n, p),(k, q)}$ as follows: If $f=\chi_{A_{(c, I, J)}}$, then

$$
\delta_{f}:=\delta_{(c, I, J)},
$$

where $\delta_{(c, I, J)}$ is defined in equation (4.4). In general, by Theorem 3.4, each element $f$ of $\left(\mathbb{Z}^{+}\right)^{\Gamma_{(n, p),(k, q)}, \succeq}$ has a unique expression as a sum

$$
f=\sum_{j=1}^{s} c_{j} \chi_{A_{j}}
$$

where $c_{j}$ are positive integers and $\emptyset \subsetneq A_{1} \subsetneq A_{2} \subsetneq \cdots \subsetneq A_{s}$ is a chain in the poset $J^{*}\left(\Gamma_{(n, p),(k, q)}\right)$. We define

$$
\delta_{f}:=\prod_{j=1}^{s} \delta_{\chi_{A_{j}}}^{c_{j}}
$$

Let

$$
\mathcal{G}_{(n, p),(k, q)}=\left\{\delta_{\chi_{A}}: A \in J^{*}\left(\Gamma_{(n, p),(k, q)}\right)\right\},
$$

and let it be given the partial ordering induced from that of $J^{*}\left(\Gamma_{(n, p),(k, q)}\right)$. Then $\delta_{f}$ is a standard monomial on $\mathcal{G}_{(n, p),(k, q)}$. We will show that the set

$$
\mathcal{B}=\left\{\delta_{f}: f \in\left(\mathbb{Z}^{+}\right)^{\Gamma_{(n, p),(k, q)}, \succeq}\right\}
$$

forms a basis for $\mathcal{L}_{(n, p),(k, q)}$. To do this, we let for each $f \in\left(\mathbb{Z}^{+}\right)^{\Gamma_{(n, p),(k, q)}, \succeq}$,

$$
m_{f}=\left(\prod_{u=1}^{k} x_{u, u}^{f_{0, i}}\right)\left(\prod_{\substack{1 \leq a \leq \min (n, k+b) \\ 1 \leq b \leq p}} y_{a, b}^{f_{b, a}-f_{b-1, a}}\right)\left(\prod_{\substack{1 \leq i \leq q \\ 1 \leq j \leq k}} z_{i, j}^{f_{-i, j}-f_{-i+1, j}}\right)
$$

where $f_{i j}$ is defined in equation (4.6).

Proposition 4.5. (i) For each $f \in\left(\mathbb{Z}^{+}\right)^{\Gamma_{(n, p),(k, q)}, \succeq}, \operatorname{LM}\left(\delta_{f}\right)=m_{f}$.

(ii) The set $\mathcal{B}$ is linearly independent. 
Proof. For (i), define $m:\left(\mathbb{Z}^{+}\right)^{\Gamma_{(n, p),(k, q)}, \succeq} \longrightarrow \mathcal{P}\left(\mathrm{M}_{n, k} \oplus \mathrm{M}_{n, p} \oplus \mathrm{M}_{q, k}\right)$ by

$$
m(f)=m_{f} .
$$

Then it is easy to check that $m$ is a semigroup isomorphism onto its image. Moreover, by Lemma 4.2,

$$
m\left(\chi_{A}\right)=\operatorname{LM}\left(\delta_{\chi_{A}}\right)
$$

for all $A \in J^{*}\left(\Gamma_{(n, p),(k, q)}\right)$.

We now let $f$ be an arbitrary element of $\left(\mathbb{Z}^{+}\right)^{\Gamma_{(n, p),(k, q)}, \succeq}$. Then $f$ can expressed uniquely as a sum

$$
f=\sum_{j=1}^{s} c_{j} \chi_{A_{j}}
$$

where $c_{j}$ are positive integers and $\emptyset \subsetneq A_{1} \subsetneq A_{2} \subsetneq \cdots \subsetneq A_{s}$ is a chain in the poset $J^{*}\left(\Gamma_{(n, p),(k, q)}\right)$. Then $\delta_{f}$ is given in equation (4.10). Since $m$ is a semigroup homomorphism,

$$
\operatorname{LM}\left(\delta_{f}\right)=\prod_{j=1}^{s} \operatorname{LM}\left(\delta_{\chi_{A_{j}}}\right)^{c_{j}}=\prod_{j=1}^{s} m\left(\chi_{A_{j}}\right)^{c_{j}}=m\left(\sum_{j=1}^{s} c_{j} \chi_{A_{j}}\right)=m(f) .
$$

This proves (i). Part (ii) now follows from the fact that the elements in $\mathcal{B}$ have distinct leading monomials.

Theorem 4.6. $\quad$ (i) The set

$$
\mathcal{B}_{(F, \alpha),(D, \beta)}=\left\{\delta_{f}: f \in \Phi\left(\mathrm{ST}_{(F, \alpha),(D, \beta)}\right)\right\}
$$

is a basis for the homogeneous component $W_{(F, \alpha),(D, \beta)}$ of $\mathcal{L}_{(n, p),(k, q)}$.

(ii) The set $\mathcal{B}$ defined in equation (4.11) is a standard monomial basis for the algebra $\mathcal{L}_{(n, p),(k, q)}$.

(iii) $\operatorname{LM}\left(\mathcal{L}_{(n, p),(k, q)}\right) \simeq\left(\mathbb{Z}^{+}\right)^{\Gamma(n, p),(k, q)}, \succeq$.

(iv) There exists a flat one-parameter family of complex algebras with general fibre $\mathcal{L}_{(n, p),(k, q)}$ and special fibre $\mathbb{C}\left[\left(\mathbb{Z}^{+}\right)^{\Gamma_{(n, p),(k, q)}, \succeq}\right]$.

Proof. We claim that if $f \in \Phi\left(\mathrm{ST}_{(F, \alpha),(D, \beta)}\right)$, then $\delta_{f} \in W_{(F, \alpha),(D, \beta)}$. This is true if $f \in \mathcal{G}_{(n, p),(k, q)}$. The general case follows from this and equation (4.3). It follows that $\mathcal{B}_{(F, \alpha),(D, \beta)} \subseteq W_{(F, \alpha),(D, \beta)}$. Moreover, $\mathcal{B}_{(F, \alpha),(D, \beta)}$ is linearly independent and the number of vectors it contains coincides with the dimension of $W_{(F, \alpha),(D, \beta)}$. So $\mathcal{B}_{(F, \alpha),(D, \beta)}$ is a basis for $W_{(F, \alpha),(D, \beta)}$ and this proves (i). Part (ii) follows from equation (4.2) and (i).

We can prove that

$$
\operatorname{LM}\left(\mathcal{L}_{(n, p),(k, q)}\right)=\left\{m_{f}: f \in\left(\mathbb{Z}^{+}\right)^{\Gamma_{(n, p),(k, q)}, \succeq}\right\}
$$

using a similar argument as in the proof of part (iii) of Theorem 3.5. Hence $\operatorname{LM}\left(\mathcal{L}_{(n, p),(k, q)}\right)$ is the range of the semigroup isomorphism $m$ defined in equation (4.12). This proves (iii). Part (iv) follows from Theorem 3.3 and the fact that $\operatorname{LM}\left(\mathcal{L}_{(n, p),(k, q)}\right)$ is finitely generated. 
4.7. The algebra $\widehat{\mathcal{L}}_{(n, p),(k, q)}$. In this subsection, we will construct the algebra $\widehat{\mathcal{L}}_{(n, p),(k, q)}$, which is a variant form of the algebra $\mathcal{L}_{(n, p),(k, q)}$. Recall that the construction of the algebra $\mathcal{L}_{(n, p),(k, q)}$ makes use of an action $\sigma$ by $\mathrm{GL}_{n} \times \mathrm{GL}_{k} \times \mathrm{GL}_{p} \times \mathrm{GL}_{q}$ on $\mathcal{P}\left(\mathrm{M}_{n, k} \oplus \mathrm{M}_{n, p} \oplus \mathrm{M}_{q, k}\right)$ given in equation (4.1). We now replace $\sigma$ by the action $\sigma^{\prime}$ defined by the formula

$$
\left(\sigma^{\prime}(g, h, a, b)(f)\right)(X, Y, R)=f\left(g^{-1} X h, g^{-1} Y a, b^{t} R h\right)
$$

where $(g, h, a, b) \in \mathrm{GL}_{n} \times \mathrm{GL}_{k} \times \mathrm{GL}_{p} \times \mathrm{GL}_{q}, f \in \mathcal{P}\left(\mathrm{M}_{n, k} \oplus \mathrm{M}_{n, p} \oplus \mathrm{M}_{q, k}\right)$ and $(X, Y, R) \in$ $\mathrm{M}_{n, k} \oplus \mathrm{M}_{n, p} \oplus \mathrm{M}_{q, k}$, and restrict $\sigma^{\prime}$ to the subgroup $\mathrm{GL}_{n} \times \mathrm{GL}_{k} \times A_{p} \times A_{q}$. Let $\widehat{\mathcal{L}}_{(n, p),(k, q)}$ be the algebra of all $U_{n} \times U_{k}$ invariants in $\mathcal{P}\left(\mathrm{M}_{n, k} \oplus \mathrm{M}_{n, p} \oplus \mathrm{M}_{q, k}\right)$ relative to the action $\sigma^{\prime}$. It is again a module for $A_{n} \times A_{k} \times A_{p} \times A_{q}$.

There is an alternative description of the algebra $\widehat{\mathcal{L}}_{(n, p),(k, q)}$. Let $\mathrm{GL}_{n} \times \mathrm{GL}_{k}$ act on $\mathcal{P}\left(\mathrm{M}_{n, k}\right)$ via the action $\tau_{n, k}^{\prime}$ defined in equation (5.3), so that, in the notation of Section 5.2, the resulting $\mathrm{GL}_{n} \times \mathrm{GL}_{k}$ module on $\mathcal{P}\left(\mathrm{M}_{n, k}\right)$ is $\mathcal{P}\left(\mathbb{C}^{n} \otimes \mathbb{C}^{k *}\right)$. Similarly, we consider the $\mathrm{GL}_{n} \times \mathrm{GL}_{p}$ module $\mathcal{P}\left(\mathbb{C}^{n} \otimes \mathbb{C}^{p *}\right)$. We also let $\mathrm{GL}_{q} \times \mathrm{GL}_{k}$ act on $\mathcal{P}\left(\mathrm{M}_{q, k}\right)$ via the action $\tau_{q, k}$ defined in equation (3.1), so the resulting module is again denoted by $\mathcal{P}\left(\mathrm{M}_{q, k}\right)$. We now form the tensor product

$$
\mathcal{P}\left(\mathbb{C}^{n} \otimes \mathbb{C}^{k *}\right) \otimes \mathcal{P}\left(\mathbb{C}^{n} \otimes \mathbb{C}^{p *}\right) \otimes \mathcal{P}\left(\mathrm{M}_{q, k}\right)
$$

which is a module for

$$
\begin{aligned}
&\left(\mathrm{GL}_{n} \times \mathrm{GL}_{k}\right) \times\left(\mathrm{GL}_{n} \times \mathrm{GL}_{p}\right) \times\left(\mathrm{GL}_{q} \times \mathrm{GL}_{k}\right) \\
& \simeq\left(\mathrm{GL}_{n} \times \mathrm{GL}_{n}\right) \times\left(\mathrm{GL}_{k} \times \mathrm{GL}_{k}\right) \times \mathrm{GL}_{p} \times \mathrm{GL}_{q},
\end{aligned}
$$

and it becomes a $\Delta\left(\mathrm{GL}_{n}\right) \times \Delta\left(\mathrm{GL}_{k}\right) \times A_{p} \times A_{q}$ module by restriction, where $\Delta\left(\mathrm{GL}_{n}\right) \cong \mathrm{GL}_{n}$ and $\Delta\left(\mathrm{GL}_{k}\right) \cong \mathrm{GL}_{k}$ embed diagonally in $\mathrm{GL}_{n} \times \mathrm{GL}_{n}$ and $\mathrm{GL}_{k} \times \mathrm{GL}_{k}$, respectively. Then

$$
\widehat{\mathcal{L}}_{(n, p),(k, q)} \simeq\left[\mathcal{P}\left(\mathbb{C}^{n} \otimes \mathbb{C}^{k *}\right) \otimes \mathcal{P}\left(\mathbb{C}^{n} \otimes \mathbb{C}^{p *}\right) \otimes \mathcal{P}\left(\mathrm{M}_{q, k}\right)\right]^{U_{n} \times U_{k}}
$$

Lemma 4.7. There is an algebra isomorphism

$$
\varphi: \mathcal{L}_{(n, p),(k, q)} \longrightarrow \widehat{\mathcal{L}}_{(n, p),(k, q)}
$$

such that

$$
\varphi(\sigma(t, s, u, v)(f))=\sigma^{\prime}\left(\left(t^{-1}\right)^{w}, s, u, v\right)(\varphi(f))
$$

for all $(t, s, u, v) \in A_{n} \times A_{k} \times A_{p} \times A_{q}$ and $f \in \mathcal{L}_{(n, p),(k, q)}$. Here, for $t=\operatorname{diag}\left(t_{1}, \ldots, t_{n}\right)$,

$$
\left(t^{-1}\right)^{w}=w\left(t^{-1}\right) w=\operatorname{diag}\left(t_{n}^{-1}, \ldots, t_{1}^{-1}\right)
$$

where

$$
w=\left(\begin{array}{rrrrr} 
& & & & 1 \\
& & & 1 & \\
& & \cdot & & \\
& \cdot & & & 0
\end{array}\right) \in \mathrm{GL}_{n} .
$$

In particular, $\varphi$ intertwines the action by $A_{k} \times A_{p} \times A_{q}$ on $\mathcal{L}_{(n, p),(k, q)}$ and $\widehat{\mathcal{L}}_{(n, p),(k, q)}$ 
Proof. Define $\varphi: \mathcal{P}\left(\mathrm{M}_{n, k} \oplus \mathrm{M}_{n, p} \oplus \mathrm{M}_{q, k}\right) \longrightarrow \mathcal{P}\left(\mathrm{M}_{n, k} \oplus \mathrm{M}_{n, p} \oplus \mathrm{M}_{q, k}\right)$ by

$$
(\varphi(f))(X, Y, R)=f(w X, w Y, R),
$$

where $f \in P$ and $(X, Y, R) \in \mathrm{M}_{n, k} \oplus \mathrm{M}_{n, p} \oplus \mathrm{M}_{q, k}$. Then one can check that the restriction of $\varphi$ to $\mathcal{L}_{(n, p),(k, q)}$ is an algebra isomorphism for $\mathcal{L}_{(n, p),(k, q)} \rightarrow \widehat{\mathcal{L}}_{(n, p),(k, q)}$ and it has the desired properties.

Remark. It would be possible to describe the Hibi cone attached to $\mathcal{L}_{(n, p)(k, q)}$ in terms of a pair of Gelfand-Tsetlin patterns whose top rows are equal. An analog expressed in the language of Young tableaux was introduced in [DRS], and was used there, and in [DeP] and [DKR] to give characteristic-free proofs of the First and Second Fundamental Theorems of invariant theory in the sense of $[\mathrm{Wy}]$. More recently, one of the present authors studied the Hibi cone attached to the space of $m$ by $n$ matrices using a combinatorial operation on Gelfand-Tsetlin patterns in [Ki3].

\section{The construction of the general iterated Pieri Algebra for GL GL $_{n}$}

The polynomial iterated Pieri rule for $\mathrm{GL}_{n}$ given in formula (3.15) describes the decomposition of the tensor product of a polynomial representation with $\ell$ representations, all of which are indexed by one-rowed Young diagrams. In this section, we shall consider the problem of decomposing the tensor product of $\mathrm{GL}_{n}$ representations of a more general form:

$$
\rho_{n}^{D, E} \otimes\left(\bigotimes_{i=1}^{p} \rho_{n}^{\left(\alpha_{i}\right)}\right) \otimes\left(\bigotimes_{j=1}^{q}\left(\rho_{n}^{\left(\beta_{j}\right)}\right)^{*}\right) .
$$

We replace the polynomial representation $\rho_{n}^{D}$ in (3.15) by any rational representation $\rho_{n}^{D, E}$, and we tensor it with $p$ representations, each of which is indexed by one-rowed Young diagram, followed by $q$ representations, each of which is dual to a representation indexed by one-rowed Young diagram. We call a description of how (5.1) decomposes a general iterated Pieri rule for $G L_{n}$.

If we wish to limit the depth of $D$ and $E$, as well as to specify the number of factors in the tensor product (5.1), we will call the description a $((k, \ell), p, q)$-Pieri rule for $\mathrm{GL}_{n}$, where $r(D) \leq k, r(E) \leq \ell$ and $k+\ell \leq n$. Thus the polynomial iterated Pieri rule given in formula $(3.15)$ is the $((k, 0), \ell, 0)$-Pieri rule. Note that we have assigned the index $\ell$ different roles in the two cases. It is an upper bound of the depth of the diagram $E$ in the $((k, \ell), p, q)$-Pieri rule, and it is the number of representations labeled by one-row Young diagrams in the tensor product in the $((k, 0), \ell, 0)$-Pieri rule.

In this section, we shall construct an algebra which encodes the general iterated Pieri rule for $\mathrm{GL}_{n}$. Later we will see that there is a close relation between the general iterated Pieri rule for $\mathrm{GL}_{n}$ and the iterated Pieri rule for $\mathrm{O}_{n}$ and for $\mathrm{Sp}_{2 n}$ discussed in [KL].

5.1. Realization of $\bigotimes_{i=1}^{p} \rho_{n}^{\left(\alpha_{i}\right)}$. Let $n$ and $p$ be positive integers, and let $\mathcal{P}\left(\mathrm{M}_{n, p}\right)$ be the algebra of polynomial functions on $\mathrm{M}_{n, p}$. We let $\mathrm{GL}_{n} \times \mathrm{GL}_{p}$ act on $\mathcal{P}\left(\mathrm{M}_{n, p}\right)$ by the action $\tau_{n, p}$ defined in equation (3.2), and we restrict this action to $\mathrm{GL}_{n} \times A_{p}$. This setting coincides 
with that of Section 3.3, with $k=0$ and $\ell=p$. So by equation (3.8), $\mathcal{P}\left(\mathrm{M}_{n, p}\right)$ decomposes as a $\mathrm{GL}_{n} \times A_{p}$ module as

$$
\mathcal{P}\left(\mathrm{M}_{n, p}\right) \simeq \sum_{\alpha=\left(\alpha_{1}, \ldots, \alpha_{p}\right) \in\left(\mathbb{Z}^{+}\right)^{p}}\left(\bigotimes_{i=1}^{p} \rho_{n}^{\left(\alpha_{i}\right)}\right) \otimes \psi_{p}^{\alpha} .
$$

We can now realize the tensor product $\bigotimes_{i=1}^{p} \rho_{n}^{\left(\alpha_{i}\right)}$ of $\mathrm{GL}_{n}$ representations as the subspace of $\mathcal{P}\left(\mathrm{M}_{n, p}\right)$ which contains functions $f$ such that $f(x a)=\psi_{p}^{\alpha}(a) f(x)$ for all $x \in \mathrm{M}_{n, p}$ and all $a \in A_{p}$. In other words, $\bigotimes_{i=1}^{p} \rho_{n}^{\left(\alpha_{i}\right)}$ can be identified with the eigenspace of $A_{p}$ in $\mathcal{P}\left(\mathrm{M}_{n, p}\right)$ corresponding to the character $\psi_{p}^{\alpha}$.

5.2. Realization of $\bigotimes_{j=1}^{q}\left(\rho_{n}^{\left(\beta_{j}\right)}\right)^{*}$. For $(g, h) \in \mathrm{GL}_{n} \times \mathrm{GL}_{q}$ and $x \in \mathrm{M}_{n, q}$, let

$$
\tau_{n, q}^{\prime}(g, h)(x)=g x h^{-1} .
$$

Under the action $\tau_{n, q}^{\prime}$,

$$
\mathrm{M}_{n, q} \simeq \mathbb{C}^{n} \otimes \mathbb{C}^{q *}
$$

and $\tau_{n, q}^{\prime}$ induces an action by $\mathrm{GL}_{n} \times \mathrm{GL}_{q}$ on $\mathcal{P}\left(\mathrm{M}_{n, q}\right)$ (we again denote it by $\tau_{n, q}^{\prime}$ ) given by

$$
\left[\tau_{n, q}^{\prime}(g, h)(f)\right](x)=f\left(g^{-1} x h\right)
$$

where $f \in \mathcal{P}\left(\mathrm{M}_{n, q}\right),(g, h) \in \mathrm{GL}_{n} \times \mathrm{GL}_{q}$ and $x \in \mathrm{M}_{n, q}$. On the other hand, $\mathrm{GL}_{n} \times \mathrm{GL}_{q}$ also acts on $\mathcal{P}\left(\mathrm{M}_{n, q}\right)$ via the action $\tau_{n, q}$ defined in (3.1). In order to distinguish these two actions on $\mathcal{P}\left(\mathrm{M}_{n, q}\right)$, we shall adopt the following conventions on notation:

(i) If $\mathrm{GL}_{n} \times \mathrm{GL}_{q}$ acts on $\mathcal{P}\left(\mathrm{M}_{n, q}\right)$ by $\tau_{n, q}$, we shall denote the corresponding module either by $\mathcal{P}\left(\mathrm{M}_{n, q}\right)$ or by $\mathcal{P}\left(\mathbb{C}^{n *} \otimes \mathbb{C}^{q *}\right)$.

(ii) If $\mathrm{GL}_{n} \times \mathrm{GL}_{q}$ acts on $\mathcal{P}\left(\mathrm{M}_{n, q}\right)$ by $\tau_{n, q}^{\prime}$, we shall denote the corresponding module only by $\mathcal{P}\left(\mathbb{C}^{n} \otimes \mathbb{C}^{q *}\right)$.

Now, as a $\mathrm{GL}_{n} \times \mathrm{GL}_{q}$ module,

$$
\mathcal{P}\left(\mathbb{C}^{n} \otimes \mathbb{C}^{q *}\right) \simeq \sum_{r(D) \leq \min (n, q)}\left(\rho_{n}^{D}\right)^{*} \otimes \rho_{q}^{D}
$$

If we restrict the action by $\mathrm{GL}_{n} \times \mathrm{GL}_{q}$ to $\mathrm{GL}_{n} \times A_{q}$, then, as in (5.2), we obtain

$$
\mathcal{P}\left(\mathbb{C}^{n} \otimes \mathbb{C}^{q *}\right) \simeq \sum_{\beta=\left(\beta_{1}, \ldots, \beta_{q}\right) \in\left(\mathbb{Z}^{+}\right)^{q}}\left(\bigotimes_{j=1}^{q}\left(\rho_{n}^{\left(\beta_{j}\right)}\right)^{*}\right) \otimes \psi_{q}^{\beta} .
$$

It follows that for each $\beta=\left(\beta_{1}, \ldots, \beta_{q}\right) \in\left(\mathbb{Z}^{+}\right)^{q}$, the tensor product $\bigotimes_{j=1}^{q}\left(\rho_{n}^{\left(\beta_{i}\right)}\right)^{*}$ of $\mathrm{GL}_{n}$ representations can be realized as the eigenspace of $A_{q}$ in $\mathcal{P}\left(\mathbb{C}^{n} \otimes \mathbb{C}^{q *}\right)$ corresponding to the character $\psi_{q}^{\beta}$. 
5.3. The algebra $\mathcal{P}\left(V_{n, k, \ell}\right)$ and realization of $\rho_{n}^{D, E}$. For $n, k, \ell \in \mathbb{Z}^{+}$, we let

$$
V_{n, k, \ell}=\mathrm{M}_{n, k} \oplus \mathrm{M}_{n, \ell}=\left\{(x, y): x \in \mathrm{M}_{n, k}, y \in \mathrm{M}_{n, \ell}\right\} .
$$

Let the group $\left(\mathrm{GL}_{n} \times \mathrm{GL}_{n}\right) \times \mathrm{GL}_{k} \times \mathrm{GL}_{\ell}$ act on $V_{n, k, \ell}$ by

$$
\left(\left(g_{1}, g_{2}\right), h_{1}, h_{2}\right) \cdot(x, y)=\left(\left(g_{1}^{-1}\right)^{t} x h_{1}^{-1}, g_{2} y h_{2}^{-1}\right),
$$

where $(x, y) \in V_{n, k, \ell},\left(g_{1}, g_{2}\right) \in \mathrm{GL}_{n} \times \mathrm{GL}_{n}, h_{1} \in \mathrm{GL}_{k}$ and $h_{2} \in \mathrm{GL}_{\ell}$. Under this action,

$$
V_{n, k, \ell} \simeq\left(\mathbb{C}^{n *} \otimes \mathbb{C}^{k *}\right) \oplus\left(\mathbb{C}^{n} \otimes \mathbb{C}^{\ell *}\right)
$$

Let $\mathcal{P}\left(V_{n, k, \ell}\right)$ be the algebra of polynomial functions on $V_{n, k, \ell}$. Then the action (5.6) induces an action of $\left(\mathrm{GL}_{n} \times \mathrm{GL}_{n}\right) \times \mathrm{GL}_{k} \times \mathrm{GL}_{\ell}$ on $\mathcal{P}\left(V_{n, k, \ell}\right)$ in the usual way, and we have

$$
\begin{aligned}
\mathcal{P}\left(V_{n, k, \ell}\right) & \simeq \mathcal{P}\left(\mathbb{C}^{n *} \otimes \mathbb{C}^{k *}\right) \otimes \mathcal{P}\left(\mathbb{C}^{n} \otimes \mathbb{C}^{\ell *}\right) \\
& \simeq \sum_{\substack{r(D) \leq \min (n, k) \\
r(E) \leq \min (n, \ell)}}\left(\rho_{n}^{D} \otimes\left(\rho_{n}^{E}\right)^{*}\right) \otimes \rho_{k}^{D} \otimes \rho_{\ell}^{E}
\end{aligned}
$$

by equations (3.3) and (5.4). By extracting the $U_{n} \times U_{n}$ invariants in equation (5.7), we obtain

$$
\mathcal{P}\left(V_{n, k, \ell}\right)^{U_{n} \times U_{n}} \simeq \sum_{\substack{r(D) \leq \min (n, k) \\ r(E) \leq \min (n, \ell)}}\left(\rho_{n}^{D}\right)^{U_{n}} \otimes\left(\rho_{n}^{E *}\right)^{U_{n}} \otimes \rho_{k}^{D} \otimes \rho_{\ell}^{E} .
$$

Since $A_{n}$ normalizes $U_{n}$, the algebra $\mathcal{P}\left(V_{n, k, \ell}\right)^{U_{n} \times U_{n}}$ is a module for $\left(A_{n} \times A_{n}\right) \times \mathrm{GL}_{k} \times \mathrm{GL}_{\ell}$. It becomes an $\Delta\left(A_{n}\right) \times \mathrm{GL}_{k} \times \mathrm{GL}_{\ell}$ module by restriction, where $\Delta\left(A_{n}\right) \cong A_{n}$ is the diagonal subgroup of $A_{n} \times A_{n}$. In the case when $k+\ell \leq n, A_{n}$ acts diagonally on the one-dimensional space $\left(\rho_{n}^{D}\right)^{U_{n}} \otimes\left(\rho_{n}^{E *}\right)^{U_{n}}$ by the character $\psi_{n}^{D, E}$, so that we have

$$
\mathcal{P}\left(V_{n, k, \ell}\right)^{U_{n} \times U_{n}} \simeq \sum_{\substack{r(D) \leq k \\ r(E) \leq \ell}} \psi_{n}^{D, E} \otimes \rho_{k}^{D} \otimes \rho_{\ell}^{E}
$$

as a $A_{n} \times \mathrm{GL}_{k} \times \mathrm{GL}_{\ell}$ module. This fact will be used in the proof of Proposition 5.1 below.

Next, we restrict the action of $\left(\mathrm{GL}_{n} \times \mathrm{GL}_{n}\right) \times \mathrm{GL}_{k} \times \mathrm{GL}_{\ell}$ on $\mathcal{P}\left(V_{n, k, \ell}\right)$ to $\Delta\left(\mathrm{GL}_{n}\right) \times$ $\mathrm{GL}_{k} \times \mathrm{GL}_{\ell}$, where $\Delta\left(\mathrm{GL}_{n}\right) \cong \mathrm{GL}_{n}$ is the diagonal subgroup of $\mathrm{GL}_{n} \times \mathrm{GL}_{n}$. Then $\mathcal{P}\left(V_{n, k, \ell}\right)$ can be decomposed as a $\mathrm{GL}_{n} \times \mathrm{GL}_{k} \times \mathrm{GL}_{\ell}$ module as in equation (5.7) except that each $\rho_{n}^{D} \otimes\left(\rho_{n}^{E}\right)^{*}$ is now an internal tensor product of $\mathrm{GL}_{n}$.

In the rest of this subsection, we shall assume that $k+\ell \leq n$ (this is called the stable range condition). Under this condition, there is another description of the $\mathrm{GL}_{n} \times \mathrm{GL}_{k} \times \mathrm{GL}_{\ell}$ module structure of $\mathcal{P}\left(V_{n, k, \ell}\right)$ which we now detail. Denote the standard coordinates on $\mathrm{M}_{n, k}$ and $\mathrm{M}_{n, \ell}$ by

$$
x=\left(x_{i j}\right) \in \mathrm{M}_{n, k} \quad \text { and } \quad y=\left(y_{i j}\right) \in \mathrm{M}_{n, \ell},
$$

and for $1 \leq a \leq k$ and $1 \leq b \leq \ell$, let

$$
r_{a b}=\sum_{i=1}^{n} x_{i a} y_{i b} \quad \text { and } \quad \Delta_{a b}=\sum_{i=1}^{n} \frac{\partial^{2}}{\partial x_{i a} \partial y_{i b}} .
$$

By the First Fundamental Theorem of Invariant Theory for $\mathrm{GL}_{n}$ ([Ho2],[GW]), the algebra $\mathcal{P}\left(V_{n, k, \ell}\right)^{\mathrm{GL}_{n}}$ of polynomials in $\mathcal{P}\left(V_{n, k, \ell}\right)$ invariant under the action by $\mathrm{GL}_{n}$ is generated by the $r_{a b}$ 's, and it is stable under the action of $\mathrm{GL}_{k} \times \mathrm{GL}_{\ell}$. Moreover, since $k+\ell \leq n$, the 
$r_{a b}$ 's are algebraically independent and $\mathcal{P}\left(V_{n, k, \ell}\right)^{\mathrm{GL}_{n}}$ can be decomposed as a $\mathrm{GL}_{k} \times \mathrm{GL}_{\ell}$ module as

$$
\mathcal{P}\left(V_{n, k, \ell}\right)^{\mathrm{GL}_{n}} \simeq \mathcal{P}\left(\mathrm{M}_{k, \ell}\right) \simeq \sum_{r(F) \leq \min (k, \ell)} \rho_{k}^{F} \otimes \rho_{\ell}^{F}
$$

Next, we let

$$
\mathcal{H}\left(V_{n, k, \ell}\right)=\left\{f \in \mathcal{P}\left(V_{n, k, \ell}\right): \Delta_{a b}(f)=0,1 \leq a \leq k, 1 \leq b \leq \ell\right\} .
$$

The space $\mathcal{H}\left(V_{n, k, \ell}\right)$ is stable under $\mathrm{GL}_{n} \times \mathrm{GL}_{k} \times \mathrm{GL}_{\ell}$, and under this action we have the following decomposition ([Ho1]):

$$
\mathcal{H}\left(V_{n, k, \ell}\right) \simeq \sum_{\substack{r(D) \leq k \\ r(E) \leq \ell}} \rho_{n}^{D, E} \otimes \rho_{k}^{D} \otimes \rho_{\ell}^{E}
$$

Let $I_{n, k, \ell}$ be the ideal of $\mathcal{P}\left(V_{n, k, \ell}\right)$ generated by $\left\{r_{a b}: 1 \leq a \leq k, 1 \leq b \leq \ell\right\}$ and form the quotient algebra $\mathcal{P}\left(V_{n, k, \ell}\right) / I_{n, k, \ell}$. Then the natural map

$$
\mathcal{H}\left(V_{n, k, \ell}\right) \longrightarrow \mathcal{P}\left(V_{n, k, \ell}\right) / I_{n, k, \ell}
$$

is a $\mathrm{GL}_{n} \times \mathrm{GL}_{k} \times \mathrm{GL}_{\ell}$ module isomorphism $([\mathrm{Ho} 1])$, so $\mathcal{P}\left(V_{n, k, \ell}\right) / I_{n, k, \ell}$ can be decomposed as in equation (5.10).

Let $\left(\mathcal{P}\left(V_{n, k, \ell}\right) / I_{n, k, \ell}\right)^{U_{k} \times U_{\ell}}$ be the algebra of $U_{k} \times U_{\ell}$ invariants in $\mathcal{P}\left(V_{n, k, \ell}\right) / I_{n, k, \ell}$. It is a module for $\mathrm{GL}_{n} \times A_{k} \times A_{\ell}$, and it follows from (5.10) that it can be decomposed as

$$
\begin{aligned}
\left(\mathcal{P}\left(V_{n, k, \ell}\right) / I_{n, k, \ell}\right)^{U_{k} \times U_{\ell}} \simeq \sum_{\substack{r(D) \leq k \\
r(E) \leq \ell}} \rho_{n}^{D, E} \otimes\left(\rho_{k}^{D}\right)^{U_{k}} \otimes\left(\rho_{\ell}^{E}\right)^{U_{\ell}} \\
\simeq \sum_{\substack{r(D) \leq k \\
r(E) \leq \ell}} \rho_{n}^{D, E} \otimes \psi_{k}^{D} \otimes \psi_{\ell}^{E} .
\end{aligned}
$$

Equation (5.12) shows that for Young diagrams $D$ and $E$ such that $r(D) \leq k$ and $r(E) \leq \ell$, we can realize the representation $\rho_{n}^{D, E}$ as the eigenspace for $A_{k} \times A_{\ell}$ in $\left(\mathcal{P}\left(V_{n, k, \ell}\right) / I_{n, k, \ell}\right)^{U_{k} \times U_{\ell}}$ corresponding to the character $\psi_{k}^{D} \times \psi_{\ell}^{E}$.

For later use, we record the following structural results on $\mathcal{P}\left(V_{n, k, \ell}\right)$.

Proposition 5.1. Assume that $k+\ell \leq n$.

(a) There is an isomorphism of $\mathrm{GL}_{n} \times \mathrm{GL}_{k} \times \mathrm{GL}_{\ell}$ modules:

$$
\mathcal{P}\left(V_{n, k, \ell}\right) \simeq \mathcal{H}\left(V_{n, k, \ell}\right) \otimes \mathcal{P}\left(V_{n, k, \ell}\right)^{\mathrm{GL}_{n}} .
$$

(b) The space $\mathcal{H}\left(V_{n, k, \ell}\right)^{U_{n}}$ of $U_{n}$ invariants in $\mathcal{H}\left(V_{n, k, \ell}\right)$ is a subalgebra of $\mathcal{P}\left(V_{n, k, \ell}\right)$, and it coincides with the algebra of $U_{n} \times U_{n}$ invariants in $\mathcal{P}\left(V_{n, k, \ell}\right)$, that is,

$$
\mathcal{H}\left(V_{n, k, \ell}\right)^{U_{n}}=\mathcal{P}\left(V_{n, k, \ell}\right)^{U_{n} \times U_{n}} .
$$

Proof. For (a), see [Ho1]. We now prove (b). By equation (5.10), we have

$$
\mathcal{H}\left(V_{n, k, \ell}\right)^{U_{n}} \simeq \sum_{\substack{r(D) \leq k \\ r(E) \leq \ell}}\left(\rho_{n}^{D, E}\right)^{U_{n}} \otimes \rho_{k}^{D} \otimes \rho_{\ell}^{E} \simeq \sum_{\substack{r(D) \leq k \\ r(E) \leq \ell}} \psi_{n}^{D, E} \otimes \rho_{k}^{D} \otimes \rho_{\ell}^{E} .
$$

Thus by equation (5.8), $\mathcal{H}\left(V_{n, k, \ell}\right)^{U_{n}}$ and $\mathcal{P}\left(V_{n, k, \ell}\right)^{U_{n} \times U_{n}}$ are isomorphic $A_{n} \times \mathrm{GL}_{k} \times \mathrm{GL}_{\ell}$ modules. Part (b) now follows from the fact that the $\mathrm{GL}_{n} \times \mathrm{GL}_{k} \times \mathrm{GL}_{\ell}$ highest weight 
vector in the submodule $\rho_{n}^{D, E} \otimes \rho_{k}^{D} \otimes \rho_{\ell}^{E}$ of $\mathcal{H}\left(V_{n, k, \ell}\right)$ is also the $\left(\mathrm{GL}_{n} \times \mathrm{GL}_{n}\right) \times \mathrm{GL}_{k} \times \mathrm{GL}_{\ell}$ highest weight vector in the submodule $\left(\rho_{n}^{D} \otimes\left(\rho_{n}^{E}\right)^{*}\right) \otimes \rho_{k}^{D} \otimes \rho_{\ell}^{E}$ of $\mathcal{P}\left(V_{n, k, \ell}\right)$.

Remark. If one writes explicitly the joint $\mathrm{GL}_{n} \times \mathrm{GL}_{k} \times \mathrm{GL}_{\ell}$ highest weight vectors in $\mathcal{H}\left(V_{n, k, \ell}\right)$, one can see by inspection that product of two of them is again harmonic. Since the space of harmonics is invariant under $\mathrm{GL}_{k} \times \mathrm{GL}_{\ell}$ and graded by the characters of $A_{n}$, this is another way of showing that $\mathcal{H}\left(V_{n, k, \ell}\right)^{U_{n}}$ is an algebra.

5.4. The iterated Pieri algebra for $\mathrm{GL}_{n}$. Let $n, k, \ell, p, q$ be positive integers such that $k+\ell \leq n$, and let $\left(\mathcal{P}\left(V_{n, k, \ell}\right) / I_{n, k, \ell}\right)^{U_{k} \times U_{\ell}}, \mathcal{P}\left(\mathbb{C}^{n *} \otimes \mathbb{C}^{p *}\right)$ and $\mathcal{P}\left(\mathbb{C}^{n} \otimes \mathbb{C}^{q *}\right)$ respectively be given the actions by $\mathrm{GL}_{n} \times A_{k} \times A_{\ell}, \mathrm{GL}_{n} \times A_{p}$ and $\mathrm{GL}_{n} \times A_{q}$ defined in Sections 5.3, 5.1 and 5.2.

We form the tensor product

$$
\mathcal{P}_{n, k, \ell, p, q}:=\left(\mathcal{P}\left(V_{n, k, \ell}\right) / I_{n, k, \ell}\right)^{U_{k} \times U_{\ell}} \otimes \mathcal{P}\left(\mathbb{C}^{n *} \otimes \mathbb{C}^{p *}\right) \otimes \mathcal{P}\left(\mathbb{C}^{n} \otimes \mathbb{C}^{q *}\right) .
$$

It is a module for

$$
\left(\mathrm{GL}_{n} \times A_{k} \times A_{\ell}\right) \times\left(\mathrm{GL}_{n} \times A_{p}\right) \times\left(\mathrm{GL}_{n} \times A_{q}\right) \simeq \mathrm{GL}_{n}^{3} \times A_{k} \times A_{\ell} \times A_{p} \times A_{q} .
$$

We restrict this action to $\Delta\left(\mathrm{GL}_{n}\right) \times A_{k} \times A_{\ell} \times A_{p} \times A_{q}$, where $\Delta\left(\mathrm{GL}_{n}\right) \cong \mathrm{GL}_{n}$ is the diagonal subgroup of $\mathrm{GL}_{n}^{3}$. Then using equations (5.12), (5.2) and (5.5), $\mathcal{P}_{n, k, \ell, p, q}$ can be decomposed as

$$
\begin{aligned}
& \left.\simeq \sum_{\substack{r(D) \leq k \\
r(E) \leq \ell}} \rho_{n}^{D, E} \otimes \psi_{k}^{D} \otimes \psi_{\ell}^{E}\right\} \otimes\left\{\sum_{\alpha \in\left(\mathbb{Z}^{+}\right)^{p}}\left(\bigotimes_{i=1}^{p} \rho_{n}^{\left(\alpha_{i}\right)}\right) \otimes \psi_{p}^{\alpha}\right\} \otimes\left\{\sum_{\beta \in\left(\mathbb{Z}^{+}\right)^{q}}\left(\bigotimes_{j=1}^{q} \rho_{n}^{\left(\beta_{j}\right) *}\right) \otimes \psi_{q}^{\beta}\right\} \\
& \simeq \sum_{\substack{r(D) \leq k, r(E) \leq \ell \\
\alpha=\left(\alpha_{i}\right) \in\left(\mathbb{Z}^{+}\right)^{p}, \beta=\left(\beta_{j}\right) \in\left(\mathbb{Z}^{+}\right)^{q}}}\left\{\rho_{n}^{D, E} \otimes\left(\bigotimes_{i=1}^{p} \rho_{n}^{\left(\alpha_{i}\right)}\right) \otimes\left(\bigotimes_{j=1}^{q} \rho_{n}^{\left(\beta_{j}\right) *}\right)\right\} \otimes \psi_{k}^{D} \otimes \psi_{\ell}^{E} \otimes \psi_{p}^{\alpha} \otimes \psi_{q}^{\beta} .
\end{aligned}
$$

For fixed $D, E, \alpha$ and $\beta$, we can realize the tensor product given in equation (5.1) as the eigenspace of $A_{k} \times A_{\ell} \times A_{p} \times A_{q}$ in $\mathcal{P}_{n, k, \ell, p, q}$ corresponding to the character $\psi_{k}^{D} \times \psi_{\ell}^{E} \times \psi_{p}^{\alpha} \times \psi_{q}^{\beta}$.

To determine how this tensor product decomposes into a sum of irreducible representations, we need to extract the $\Delta\left(\mathrm{GL}_{n}\right)$ highest weight vectors in this space. We will abuse notation and denote the standard maximal unipotent subgroup of $\Delta\left(\mathrm{GL}_{n}\right)$ also by $U_{n}$. Thus we consider the subalgebra of $U_{n}$ invariants in $\mathcal{P}_{n, k, \ell, p, q}$ :

$$
\mathfrak{A}_{n, k, \ell, p, q}:=\mathcal{P}_{n, k, \ell, p, q}^{U_{n}} .
$$

The algebra $\mathfrak{A}_{n, k, \ell, p, q}$ is a module for $\mathbf{A}_{n, k, \ell, p, q}=A_{n} \times A_{k} \times A_{\ell} \times A_{p} \times A_{q}$. In particular, it is a multi-graded algebra with grading given by the characters of $\mathbf{A}_{n, k, \ell, p, q}$. Let $\mathcal{E}_{(G, F),(D, E), \alpha, \beta}$ be the eigenspace of $\mathbf{A}_{n, k, \ell, p, q}$ corresponding to the eigencharacter

$$
\psi_{n}^{G, F} \times \psi_{k}^{D} \times \psi_{\ell}^{E} \times \psi_{p}^{\alpha} \times \psi_{q}^{\beta} .
$$


Then $\mathcal{E}_{(G, F),(D, E), \alpha, \beta}$ can be identified with the space of $\mathrm{GL}_{n}$ highest weight vectors of weight $\psi_{n}^{G, F}$ in the tensor product given in equation (5.1), so that

$$
\operatorname{dim} \mathcal{E}_{(G, F),(D, E), \alpha, \beta}=\operatorname{dim} \operatorname{Hom}_{\mathrm{GL}}\left(\rho_{n}^{G, F}, \rho_{n}^{D, E} \otimes\left(\bigotimes_{i=1}^{p} \rho_{n}^{\left(\alpha_{i}\right)}\right) \otimes\left(\bigotimes_{j=1}^{q} \rho_{n}^{\left(\beta_{j}\right) *}\right)\right),
$$

which is the multiplicity of $\rho_{n}^{G, F}$ in the tensor product (5.1). Therefore, the algebra structure of $\mathfrak{A}_{n, k, \ell, p, q}$ encodes information on the decomposition of tensor products of the form (5.1). In view of this property, we call $\mathfrak{A}_{n, k, \ell, p, q}$ a general iterated Pieri algebra for $\mathrm{GL}_{n}$. When it may be helpful, we refine our terminology according to the comments at the beginning of this section, and may call this algebra the $((k, \ell), p, q)$-iterated Pieri algebra for $\mathrm{GL}_{n}$.

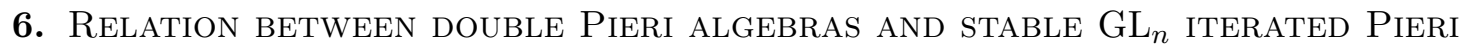 ALGEBRAS}

In Section 5.4 we defined the general iterated Pieri algebra $\mathfrak{A}_{n, k, \ell, p, q}$ for $\mathrm{GL}_{n}$ for positive integers $n, k, \ell, p, q$. In this section, we shall focus on the case when $n \geq k+p+\ell+q$, and we call the corresponding general iterated Pieri algebra stable. We shall prove that the stable $\mathrm{GL}_{n}$ iterated Pieri algebras $\mathfrak{A}_{n, k, \ell, p, q}$ is isomorphic to the tensor product of $\mathcal{L}_{(n, p),(k, q)}$, $\widehat{\mathcal{L}}_{(n, q),(\ell, p)}$ and a polynomial algebra. Using this and the results in Section 4 , we deduce that $\mathfrak{A}_{n, k, \ell, p, q}$ has a standard monomial basis.

6.1. Stable $\mathrm{GL}_{n}$ iterated Pieri algebras. We shall show that the stable $\mathrm{GL}_{n}$ iterated Pieri algebras $\mathfrak{A}_{n, k, \ell, p, q}$ can be identified with a subalgebra of a polynomial algebra, and from which we deduce the following theorem.

Theorem 6.1. (Relation with the $\mathrm{GL}_{n}$ iterated Pieri algebras) If $n \geq k+p+\ell+q$, then there is an isomorphism

$$
\mathfrak{A}_{n, k, \ell, p, q} \simeq \mathcal{L}_{(n, p),(k, q)} \otimes \widehat{\mathcal{L}}_{(n, q),(\ell, p)} \otimes \mathcal{P}\left(\mathrm{M}_{p, q}\right)
$$

of algebras and $A_{n} \times A_{k} \times A_{\ell} \times A_{p} \times A_{q}$ modules.

Proof. For $(X, Y) \in V_{n, k+p, \ell+q}=\mathrm{M}_{n, k+p} \oplus \mathrm{M}_{n, \ell+q}$, let $\pi(X, Y)=\left(X_{0}, Y_{0}\right) \in V_{n, k, \ell}=$ $\mathrm{M}_{n, k} \oplus \mathrm{M}_{n, \ell}$ where $X_{0}$ (resp. $Y_{0}$ ) is obtained from $X$ (resp. $Y$ ) by removing its last $p$ columns (resp. $q$ columns). The map $\pi: V_{n, k+p, \ell+q} \rightarrow V_{n, k, \ell}$ is a projection, and it induces an injection $\pi^{*}: \mathcal{P}\left(V_{n, k, \ell}\right) \hookrightarrow \mathcal{P}\left(V_{n, k+p, \ell+q}\right)$ in the usual way. We shall identify $\mathcal{P}\left(V_{n, k, \ell}\right)$ with its image under $\pi^{*}$ in $\mathcal{P}\left(V_{n, k+p, \ell+q}\right)$.

Let $\tilde{I}_{n, k, \ell}$ be the ideal in $\mathcal{P}\left(V_{n, k+p, \ell+q}\right)$ generated by $I_{n, k, \ell}$ (cf. (5.11)). Then by equations (5.13) and (5.14), we have

$$
\begin{aligned}
\mathfrak{A}_{n, k, \ell, p, q}=\mathcal{P}_{n, k, \ell, p, q}^{U_{n}} & \simeq\left(\left(\mathcal{P}\left(V_{n, k, \ell}\right) / I_{n, k, \ell}\right)^{U_{k} \times U_{\ell}} \otimes \mathcal{P}\left(\mathbb{C}^{n *} \otimes \mathbb{C}^{p *}\right) \otimes \mathcal{P}\left(\mathbb{C}^{n} \otimes \mathbb{C}^{q *}\right)\right)^{U_{n}} \\
& \simeq\left(\mathcal{P}\left(V_{n, k+p, \ell+q}\right) / \tilde{I}_{n, k, \ell}\right)^{U_{n} \times U_{k} \times U_{\ell}} .
\end{aligned}
$$

Now the algebra of $\mathrm{GL}_{n}$ invariants in $\mathcal{P}\left(V_{n, k+p, \ell+q}\right)$ is generated by

$$
\left\{r_{i j}: 1 \leq i \leq k+p, 1 \leq j \leq \ell+q\right\}
$$


where $r_{i j}$ are the basic invariants given in equation (5.9). As a block matrix we write

$$
\begin{aligned}
\left(r_{i j}\right)_{(k+p) \times(\ell+q)} & =\left(x_{c i}\right)_{n \times(k+p)}^{t}\left(y_{c j}\right)_{n \times(\ell+q)} \\
& =\left[\begin{array}{ll}
R_{0} & R_{1} \\
R_{2} & R_{3}
\end{array}\right]_{(k+p) \times(\ell+q)}
\end{aligned}
$$

and let $W_{i}$ be the space spanned by the entries of $R_{i}$

$$
\begin{aligned}
& W_{0}=\operatorname{Span}\left\{r_{i j}: 1 \leq i \leq k, 1 \leq j \leq \ell\right\} \\
& W_{1}=\operatorname{Span}\left\{r_{i j}: 1 \leq i \leq k, \ell+1 \leq j \leq \ell+q\right\} \\
& W_{2}=\operatorname{Span}\left\{r_{i j}: k+1 \leq i \leq k+p, 1 \leq j \leq \ell\right\} \\
& W_{3}=\operatorname{Span}\left\{r_{i j}: k+1 \leq i \leq k+p, \ell+1 \leq j \leq \ell+q\right\} .
\end{aligned}
$$

Now we let $\mathbb{C}\left[W_{0}\right], \mathbb{C}\left[W_{1}\right], \mathbb{C}\left[W_{2}\right]$ and $\mathbb{C}\left[W_{3}\right]$ be the algebras generated by $W_{0}, W_{1}, W_{2}$ and $W_{3}$, respectively. The algebras $\mathbb{C}\left[W_{0}\right], \mathbb{C}\left[W_{1}\right], \mathbb{C}\left[W_{2}\right]$ and $\mathbb{C}\left[W_{3}\right]$ are modules for $\mathrm{GL}_{k} \times \mathrm{GL}_{\ell}$, $\mathrm{GL}_{q} \times \mathrm{GL}_{k}, \mathrm{GL}_{p} \times \mathrm{GL}_{\ell}$, and $\mathrm{GL}_{p} \times \mathrm{GL}_{q}$ respectively, and under these actions,

$$
\begin{array}{lll}
\mathbb{C}\left[W_{0}\right] \simeq \mathcal{P}\left(\mathrm{M}_{k, \ell}\right), & \mathbb{C}\left[W_{1}\right] \simeq \mathcal{P}\left(\mathrm{M}_{q, k}\right), \\
\mathbb{C}\left[W_{2}\right] \simeq \mathcal{P}\left(\mathrm{M}_{p, \ell}\right), & \mathbb{C}\left[W_{3}\right] \simeq \mathcal{P}\left(\mathrm{M}_{p, q}\right) .
\end{array}
$$

See Section 5.2 for notations. Recall from equation (5.10) that the space $\mathcal{H}\left(V_{n, k+p, \ell+q}\right)$ of $\mathrm{GL}_{n}$ harmonic polynomials in $\mathcal{P}\left(V_{n, k+p, \ell+q}\right)$ carries an action by $\mathrm{GL}_{n} \times \mathrm{GL}_{k+p} \times \mathrm{GL}_{\ell+q}$, so it becomes a $\mathrm{GL}_{n} \times \mathrm{GL}_{k} \times \mathrm{GL}_{p} \times \mathrm{GL}_{\ell} \times \mathrm{GL}_{q}$ module by restriction. We now form the tensor product

$$
\mathcal{H}\left(V_{n, k+p, \ell+q}\right) \otimes \mathbb{C}\left[W_{1}\right] \otimes \mathbb{C}\left[W_{2}\right] \otimes \mathbb{C}\left[W_{3}\right]
$$

which is a module for $\mathrm{GL}_{n} \times \mathrm{GL}_{k} \times \mathrm{GL}_{\ell} \times \mathrm{GL}_{p} \times \mathrm{GL}_{q}$.

Since $n \geq(k+p)+(\ell+q)$, by part (a) of Proposition 5.1,

$$
\mathcal{P}\left(V_{n, k+p, \ell+q}\right) \simeq \mathcal{H}\left(V_{n, k+p, \ell+q}\right) \otimes \mathcal{P}\left(V_{n, k+p, \ell+q}\right)^{\mathrm{GL}_{n}}
$$

as $\mathrm{GL}_{n} \times \mathrm{GL}_{k+p} \times \mathrm{GL}_{\ell+q}$ modules. For $h \in \mathcal{H}\left(V_{n, k+p, \ell+q}\right), f_{1} \in \mathbb{C}\left[W_{1}\right], f_{2} \in \mathbb{C}\left[W_{2}\right]$ and $f_{3} \in \mathbb{C}\left[W_{3}\right]$, we define

$$
\begin{aligned}
\phi: \mathcal{H}\left(V_{n, k+p, \ell+q}\right) \otimes \mathbb{C}\left[W_{1}\right] \otimes \mathbb{C}\left[W_{2}\right] \otimes \mathbb{C}\left[W_{3}\right] & \rightarrow \mathcal{P}\left(V_{n, k+p, \ell+q}\right) / \tilde{I}_{n, k, \ell} \\
\phi\left(h \otimes f_{1} \otimes f_{2} \otimes f_{3}\right) & =h f_{1} f_{2} f_{3}+\tilde{I}_{n, k, \ell}
\end{aligned}
$$

Then by equation (6.1), $\phi$ can be extended to a $\mathrm{GL}_{n} \times \mathrm{GL}_{k} \times \mathrm{GL}_{\ell} \times \mathrm{GL}_{p} \times \mathrm{GL}_{q}$ module isomorphism for

$$
\mathcal{H}\left(V_{n, k+p, \ell+q}\right) \otimes \mathbb{C}\left[W_{1}\right] \otimes \mathbb{C}\left[W_{2}\right] \otimes \mathbb{C}\left[W_{3}\right] \simeq \mathcal{P}\left(V_{n, k+p, \ell+q}\right) / \tilde{I}_{n, k, \ell}
$$


It follows from this and part (b) of Proposition 5.1 (in the fourth equivalence below) that

$$
\begin{aligned}
& \mathfrak{A}_{n, k, \ell, p, q} \\
\simeq & \left(\mathcal{P}\left(V_{n, k+p, \ell+q}\right) / \tilde{I}_{n, k, \ell}\right)^{U_{n} \times U_{k} \times U_{\ell}} \\
\simeq & {\left[\mathcal{H}\left(V_{n, k+p, \ell+q}\right) \otimes \mathbb{C}\left[W_{1}\right] \otimes \mathbb{C}\left[W_{2}\right] \otimes \mathbb{C}\left[W_{3}\right]\right]^{U_{n} \times U_{k} \times U_{\ell}} } \\
\simeq & {\left[\mathcal{H}\left(V_{n, k+p, \ell+q}\right)^{U_{n}} \otimes \mathcal{P}\left(\mathrm{M}_{q, k}\right) \otimes \mathcal{P}\left(\mathrm{M}_{p, \ell}\right)\right]^{U_{k} \times U_{\ell}} \otimes \mathcal{P}\left(\mathrm{M}_{p, q}\right) } \\
\simeq & {\left[\mathcal{P}\left(V_{n, k+p, \ell+q}\right)^{U_{n} \times U_{n}} \otimes \mathcal{P}\left(\mathrm{M}_{q, k}\right) \otimes \mathcal{P}\left(\mathrm{M}_{p, \ell}\right)\right]^{U_{k} \times U_{\ell}} \otimes \mathcal{P}\left(\mathrm{M}_{p, q}\right)(\text { by Prop. 5.1(b)) }} \\
\simeq & {\left[\mathcal{P}\left(\mathrm{M}_{n, k+p}\right)^{U_{n}} \otimes \mathcal{P}\left(\mathbb{C}^{n} \otimes \mathbb{C}^{(\ell+q) *}\right)^{U_{n}} \otimes \mathcal{P}\left(\mathrm{M}_{q, k}\right) \otimes \mathcal{P}\left(\mathrm{M}_{p, \ell}\right)\right]^{U_{k} \times U_{\ell}} \otimes \mathcal{P}\left(\mathrm{M}_{p, q}\right) } \\
\simeq & {\left[\mathcal{P}\left(\mathrm{M}_{n, k+p}\right) \otimes \mathcal{P}\left(\mathbb{C}^{n} \otimes \mathbb{C}^{(\ell+q) *}\right) \otimes \mathcal{P}\left(\mathrm{M}_{q, k}\right) \otimes \mathcal{P}\left(\mathrm{M}_{p, \ell}\right)\right]^{U_{n} \times U_{n} \times U_{k} \times U_{\ell}} \otimes \mathcal{P}\left(\mathrm{M}_{p, q}\right) } \\
\simeq & {\left[\mathcal{P}\left(\mathrm{M}_{n, k}\right) \otimes \mathcal{P}\left(\mathrm{M}_{n, p}\right) \otimes \mathcal{P}\left(\mathbb{C}^{n} \otimes \mathbb{C}^{\ell *}\right) \otimes \mathcal{P}\left(\mathbb{C}^{n} \otimes \mathbb{C}^{q *}\right)\right.} \\
\simeq & \left\{\left[\mathcal{P}\left(\mathrm{M}_{n, k}\right) \otimes \mathcal{P}\left(\mathrm{M}_{n, p}\right) \otimes \mathcal{P}\left(\mathrm{M}_{q, k}\right)\right]\right. \\
& \left.\otimes\left[\mathcal{P}\left(\mathbb{C}^{n} \otimes \mathbb{C}^{\ell *}\right) \otimes \mathcal{P}\left(\mathbb{C}^{n} \otimes \mathbb{C}^{q *}\right) \otimes \mathcal{P}\left(\mathrm{M}_{p, \ell}\right)\right]\right\}^{U_{n} \times U_{n} \times U_{k} \times U_{\ell}} \otimes \mathcal{P}\left(\mathrm{M}_{p, q}\right) \\
\simeq & {\left[\mathcal{P}\left(\mathrm{M}_{n, k}\right) \otimes \mathcal{P}\left(\mathrm{M}_{n, p}\right) \otimes \mathcal{P}\left(\mathrm{M}_{q, k}\right)\right]^{U_{n} \times U_{k}} } \\
& \quad \otimes\left[\mathcal{P}\left(\mathbb{C}^{n} \otimes \mathbb{C}^{\ell *}\right) \otimes \mathcal{P}\left(\mathbb{C}^{n} \otimes \mathbb{C}^{q *}\right) \otimes \mathcal{P}\left(\mathrm{M}_{p, \ell}\right)\right]^{U_{n} \times U_{\ell}} \otimes \mathcal{P}\left(\mathrm{M}_{p, q}\right) \\
\simeq & \mathcal{L}(n, p),(k, q) \otimes \widehat{\mathcal{L}}_{(n, q),(\ell, p)} \otimes \mathcal{P}\left(\mathrm{M}_{p, q}\right) .
\end{aligned}
$$

6.2. Standard monomial basis for $\mathfrak{A}_{n, k, \ell, p, q}$. Assume that $n \geq k+p+\ell+q$. By Proposition 6.1 and Lemma 4.7, we have an algebra isomorphism

$$
\begin{aligned}
\mathfrak{A}_{n, k, \ell, p, q} & \simeq \mathcal{L}_{(n, p),(k, q)} \otimes \widehat{\mathcal{L}}_{(n, q),(\ell, p)} \otimes \mathcal{P}\left(\mathrm{M}_{p, q}\right) \\
& \simeq \mathcal{L}_{(n, p),(k, q)} \otimes \mathcal{L}_{(n, q),(\ell, p)} \otimes \mathcal{P}\left(\mathrm{M}_{p, q}\right) .
\end{aligned}
$$

Thus a choice of a basis in each of $\mathcal{L}_{(n, p),(k, q)}, \mathcal{L}_{(n, q),(\ell, p)}$ and $\mathcal{P}\left(\mathrm{M}_{p, q}\right)$ will determine a basis for $\mathfrak{A}_{n, k, \ell, p, q}$. Now by Theorem 4.6, $\mathcal{B}_{(n, p),(k, q)}=\left\{\delta_{f}: f \in\left(\mathbb{Z}^{+}\right)^{\Gamma_{(n, p),(k, q)}, \succeq}\right\}$ is a basis for $\mathcal{L}_{(n, p),(k, q)}$ and $\mathcal{B}_{(n, q),(\ell, p)}=\left\{\delta_{g}: g \in\left(\mathbb{Z}^{+}\right)^{\Gamma_{(n, q),(\ell, p)}, \succeq}\right\}$ is a basis for $\mathcal{L}_{(n, q),(\ell, p)}$. On the other hand, let $\left(u_{i j}\right)$ be the standard coordinates on $\mathrm{M}_{p, q}$, so that $\mathcal{P}\left(\mathrm{M}_{p, q}\right)$ can be regarded as a polynomial algebra on the $u_{i j}$. So a basis for $\mathcal{P}\left(\mathrm{M}_{p, q}\right)$ is given by the set of all monomials in $u_{i j}$, that is, $\left\{u^{\alpha}: \alpha \in\left(\mathbb{Z}^{+}\right)^{p q}\right\}$, where for each $\alpha=\left(\alpha_{i j}\right) \in\left(\mathbb{Z}^{+}\right)^{p q}, u^{\alpha}=\prod_{i, j} u_{i j}^{\alpha_{i j}}$. We now let

$$
\widetilde{\Omega}=\left(\mathbb{Z}^{+}\right)^{\Gamma_{(n, p),(k, q)}, \succeq} \times\left(\mathbb{Z}^{+}\right)^{\Gamma_{(n, q),(\ell, p)}, \succeq} \times\left(\mathbb{Z}^{+}\right)^{p q}
$$

and

$$
\widetilde{\mathcal{B}}=\left\{\delta_{f} \otimes \delta_{g} \otimes u^{\alpha}:(f, g, \alpha) \in \widetilde{\Omega}\right\} .
$$

Then $\widetilde{\mathcal{B}}$ is a basis for $\mathfrak{A}_{n, k, \ell, p, q}$. In fact, $\widetilde{\Omega}$ is a Hibi cone and $\widetilde{\mathcal{B}}$ is the associated standard monomial basis. Moreover, the algebra $\mathfrak{A}_{n, k, \ell, p, q}$ can be flatly deformed to the semigroup algebra $\mathbb{C}[\widetilde{\Omega}]$, where

$$
\mathbb{C}[\widetilde{\Omega}] \simeq \mathbb{C}\left[\left(\mathbb{Z}^{+}\right)^{\Gamma_{(n, p),(k, q)}, \succeq}\right] \otimes \mathbb{C}\left[\left(\mathbb{Z}^{+}\right)^{\Gamma_{(n, q),(\ell, p)}, \succeq}\right] \otimes \mathcal{P}\left(\mathrm{M}_{p, q}\right) .
$$


The poset $\widetilde{\Gamma}$ associated with $\widetilde{\Omega}$ is constructed as follows: Recall that for posets $P$ and $Q$ on disjoint sets, the disjoint union $P \dot{\cup} Q$ is the poset on their union such that $x \leq y$ in $P \dot{\cup} Q$ if either $x, y \in P$ and $x \leq y$ in $P$, or $x, y \in Q$ and $x \leq y$ in $Q$. Then

$$
\widetilde{\Gamma}=\Gamma_{(n, p),(k, q)} \dot{\cup} \Gamma_{(n, q),(\ell, p)} \dot{\cup} \Gamma_{p, q}
$$

where $\Gamma_{p, q}$ is the disjoint union of $p q$ singleton sets, and it is straightforward to see that

$$
\widetilde{\Omega}=\left(\mathbb{Z}^{+}\right)^{\widetilde{\Gamma}} \succeq .
$$

(see [DaP, Sections 1.32 and 5.15].)

\section{7. $\mathrm{O}_{n}$ AND $\mathrm{Sp}_{2 n}$ Stable iterated Pieri algebras}

The stable iterated Pieri algebras for $\mathrm{O}_{n}$ and $\mathrm{Sp}_{2 n}$ were first introduced in the paper [KL]. It was pointed out there that the stable iterated Pieri algebras for $\mathrm{O}_{2 n}$ and for $\mathrm{Sp}_{2 n}$ are isomorphic, so we only need to consider the iterated Pieri algebra for $\mathrm{O}_{n}$. Such an algebra is specified by 3 parameters $n, k$ and $p$, and is denoted by $\mathcal{A}_{n, k, p}$. In this section, we shall show that $\mathcal{A}_{n, k, p}$ can also be described in terms of the double Pieri algebra $\mathcal{L}_{(n, p),(k, p)}$. Precisely, $\mathcal{A}_{n, k, p}$ is isomorphic to the tensor product of $\mathcal{L}_{(n, p),(k, p)}$ and the polynomial algebra on $\Lambda^{2}\left(\mathbb{C}^{p}\right)$, the second exterior power of $\mathbb{C}^{p}$. We shall also describe a relationship between $\mathcal{A}_{n, k, p}$ and the stable $\mathrm{GL}_{n}$ iterated algebra $\mathfrak{A}_{n, k, 0, p, p}$.

We first briefly recall the construction of $\mathcal{A}_{n, k, p}$ given in [KL]. Let $k$ and $p$ be positive integers such that $2(k+p)<n$. Let $\mathrm{O}_{n} \times \mathrm{GL}_{k}$ act on $\mathcal{P}\left(\mathrm{M}_{n, k}\right)$ by the formula

$$
((g, h) f)(X)=f\left(g^{-1} X h\right) .
$$

where $(g, h) \in \mathrm{O}_{n} \times \mathrm{GL}_{k}, f \in \mathcal{P}\left(\mathrm{M}_{n, k}\right)$ and $X \in \mathrm{M}_{n, k}$, and let $I_{n k}$ be the ideal of $\mathcal{P}\left(\mathrm{M}_{n, k}\right)$ generated by all the $\mathrm{O}_{n}$ invariants in $\mathcal{P}\left(\mathrm{M}_{n, k}\right)$ with positive degree. Then the action (7.1) induces an action by $\mathrm{O}_{n} \times \mathrm{GL}_{k}$ on the quotient algebra $\mathcal{P}\left(\mathrm{M}_{n, k}\right) / I_{n k}$, and under this action, we have $([\mathrm{Ho} 2])$

$$
\mathcal{P}\left(\mathrm{M}_{n, k}\right) / I_{n k} \simeq \sum_{r(D) \leq k} \sigma_{n}^{D} \otimes \rho_{k}^{D} .
$$

Here $\sigma_{n}^{D}$ is the irreducible representation of $\mathrm{O}_{n}$ associated with the Young diagram $D$ (cf. Section 2.2). By extracting $U_{k}$ invariants in (7.2), we obtain

$$
\left(\mathcal{P}\left(\mathrm{M}_{n, k}\right) / I_{n k}\right)^{U_{k}} \simeq \sum_{r(D) \leq k} \sigma_{n}^{D} \otimes\left(\rho_{k}^{D}\right)^{U_{k}}
$$

Next, we repeat this construction with $k=1$. For each $1 \leq j \leq p$, let $\mathbb{C}_{j}^{n}$ be a copy of $\mathbb{C}^{n}$, and let $I_{n 1}^{(j)}$ be the ideal of $\mathcal{P}\left(\mathbb{C}_{j}^{n}\right)$ generated by all the $\mathrm{O}_{n}$ invariants in $\mathcal{P}\left(\mathbb{C}_{j}^{n}\right)$ with positive degree. Then under the action of $\mathrm{O}_{n} \times \mathrm{GL}_{1}$, we have

$$
\mathcal{P}\left(\mathbb{C}_{j}^{n}\right) / I_{n 1}^{(j)} \simeq \sum_{\alpha_{j} \geq 0} \sigma_{n}^{\left(\alpha_{j}\right)} \otimes \rho_{1}^{\left(\alpha_{j}\right)}
$$

We now form the tensor product of algebras

$$
\mathfrak{T}_{n, k, p}:=\left(\mathcal{P}\left(\mathrm{M}_{n, k}\right) / I_{n k}\right)^{U_{k}} \otimes\left\{\bigotimes_{j=1}^{p}\left(\mathcal{P}\left(\mathbb{C}_{j}^{n}\right) / I_{n 1}^{(j)}\right)\right\} .
$$


It is a module for $\mathrm{O}_{n} \times A_{k} \times \mathrm{GL}_{1}^{p} \simeq \mathrm{O}_{n} \times A_{k} \times A_{p}$, where we identify $\mathrm{GL}_{1}^{p}$ with the diagonal torus $A_{p}$ of $\mathrm{GL}_{p}$. Then as a $\mathrm{O}_{n} \times A_{k} \times A_{p}$ module,

$$
\begin{aligned}
\mathfrak{T}_{n, k, p} & \simeq\left(\sum_{r(D) \leq k} \sigma_{n}^{D} \otimes\left(\rho_{k}^{D}\right)^{U_{k}}\right) \otimes\left\{\bigotimes_{j=1}^{p}\left(\sum_{\alpha_{j} \geq 0} \sigma_{n}^{\left(\alpha_{j}\right)} \otimes \rho_{1}^{\left(\alpha_{j}\right)}\right)\right\} \\
& \simeq \sum_{\substack{r(D) \leq k \\
\alpha=\left(\alpha_{1}, \ldots, \alpha_{p}\right) \in\left(\mathbb{Z}^{+}\right)^{p}}}\left\{\sigma_{n}^{D} \otimes\left(\bigotimes_{j=1}^{p} \sigma_{n}^{\left(\alpha_{j}\right)}\right)\right\} \otimes \psi_{k}^{D} \otimes \psi_{p}^{\alpha} .
\end{aligned}
$$

For a fixed Young diagram $D$ with $r(D) \leq k$ and a fixed $\alpha=\left(\alpha_{1}, \ldots, \alpha_{p}\right) \in\left(\mathbb{Z}^{+}\right)^{p}$, the $\psi_{k}^{D} \times \psi_{p}^{\alpha}$-eigenspace of $A_{k} \times A_{p}$ in $\mathfrak{T}_{n, k, p}$ is a copy of the tensor product

$$
\sigma_{n}^{D} \otimes\left(\bigotimes_{j=1}^{p} \sigma_{n}^{\left(\alpha_{j}\right)}\right) \text {. }
$$

We now let

$$
\mathcal{A}_{n, k, p}:=\mathfrak{T}_{n, k, p}{ }^{U_{\mathrm{SO}}}
$$

be the algebra of $U_{\mathrm{SO}_{n}}$ invariants in $\mathfrak{T}_{n, k, p}$, which is a module for $A_{n} \times A_{k} \times A_{p}$. It carries a multigrading, and each of its homogeneous components can be identified with the space of $\mathrm{SO}_{n}$ highest weight vectors of a certain weight in a tensor product of the form (7.3). See $[\mathrm{KL}]$ for a detailed explanation.

Since $2(k+p)<n$, the $\mathrm{O}_{n}$ irreducible constituents in the tensor product (7.3) are determined by the $\mathrm{SO}_{n}$ highest weight vectors they contain. Thus, the multiplicity of an irreducible representation of $\mathrm{O}_{n}$ in this tensor product coincides with the dimension of the corresponding homogeneous component of $\mathcal{A}_{n, k, p}$. In this sense, the algebra structure of $\mathcal{A}_{n, k, p}$ encodes the iterated Pieri rule for $\mathrm{O}_{n}$. In view of this property, we call the algebra $\mathcal{A}_{n, k, p}$ a stable iterated Pieri algebra for $\mathrm{O}_{n}$.

Proposition 7.1. [KL, Proposition 4.1] For $2(k+p)<n$, the iterated Pieri algebra $\mathcal{A}_{n, k, p}$ for $\mathrm{O}_{n}$ is isomorphic as an algebra and $A_{n} \times A_{k} \times A_{p}$ module to

$$
\left(\mathcal{P}\left(\mathrm{M}_{n, k}\right) \otimes \mathcal{P}\left(\mathrm{M}_{n, p}\right) \otimes \mathcal{P}\left(\mathrm{M}_{k, p}\right)\right)^{U_{n} \times U_{k}} \otimes \mathcal{P}\left(\wedge^{2}\left(\mathbb{C}^{p}\right)\right),
$$

where $\wedge^{2}\left(\mathbb{C}^{p}\right)$ is the second exterior power of $\mathbb{C}^{p}$.

Then, it follows at once from Proposition 7.1 that:

Theorem 7.2. (Relation with the $\mathrm{O}_{n}$ iterated Pieri algebras) If $2(k+p)<n$, then we have the isomorphism

$$
\mathcal{A}_{n, k, p} \simeq \mathcal{L}_{(n, p),(k, p)} \otimes \mathcal{P}\left(\wedge^{2}\left(\mathbb{C}^{p}\right)\right)
$$

of algebras and $A_{n} \times A_{k} \times A_{p}$ modules.

Corollary 7.3. (Relationship between $\mathrm{GL}_{n}$ and $\mathrm{O}_{n}$ iterated Pieri algebras) If $2(k+p)<n$, then we have the isomorphism

$$
\mathfrak{A}_{n, k, 0, p, p} \simeq \mathcal{A}_{n, k, p} \otimes \mathcal{P}\left(S^{2}\left(\mathbb{C}^{p}\right)\right)
$$

of algebras and $A_{n} \times A_{k} \times A_{p}$ modules, where $S^{2}\left(\mathbb{C}^{p}\right)$ is the symmetric square of $\mathbb{C}^{p}$. So $\mathcal{A}_{n, k, p}$ can be identified as a subalgebra and a quotient of $\mathfrak{A}_{n, k, 0, p, p}$. 
Proof. If $\ell=0$ and $p=q$, then by Proposition 6.1,

$$
\begin{aligned}
\mathfrak{A}_{n, k, 0, p, p} & \simeq \mathcal{L}_{(n, p),(k, p)} \otimes \mathcal{P}\left(\mathrm{M}_{p, p}\right) \\
& \simeq \mathcal{L}_{(n, p),(k, p)} \otimes \mathcal{P}\left(\wedge^{2}\left(\mathbb{C}^{p}\right)\right) \otimes \mathcal{P}\left(S^{2}\left(\mathbb{C}^{p}\right)\right) \\
& \simeq \mathcal{A}_{n, k, p} \otimes \mathcal{P}\left(S^{2}\left(\mathbb{C}^{p}\right)\right)
\end{aligned}
$$

\section{Acknowledgments}

We are grateful to the Institute for Advanced Studies and the Department of Mathematics of Hong Kong University of Science and Technology, the Institute of Mathematical Sciences at the National University of Singapore and the Max Planck Institute of Bonn, Germany, for providing a productive working atmosphere.

\section{REFERENCES}

[AB] V. Alexeev and M. Brion, Toric degenerations of spherical varieties, Selecta Math. (N.S.) 10 (2004), 453-478.

[BH] W. Bruns and J. Herzog, Cohen-Macaulay Rings, Cambridge Univ. Press, 1998, 453 pp.

[Bu] A. S. Buch, The saturation conjecture (after A. Knutson and T. Tao). With an appendix by William Fulton. Enseign. Math. (2) 46 (2000), no. 1-2, 43-60.

[Ca] P. Caldero, Toric degenerations of Schubert varieties, Transf. Groups 7 (2002), No. 1, 51-60.

[Ch] Chirivi, R. LS algebras and application to Schubert varieties. Transform. Groups 5 (2000), no. 3, 245-264.

[CHV] A. Conca, J. Herzog and G. Valla, SAGBI basis with applications to blow-up algebras, J. Reine Angew. Math. 474 (1996), 113-138.

[CLO] D. Cox, J. Little and D. O'Shea, Ideals, Varieties, and Algorithms. An Introduction to Computational Algebraic Geometry and Commutative Algebra, Second edition, Undergraduate Texts in Mathematics, Springer-Verlag, New York, 1997.

[DK] P. Doolan and S. Kim, The Littlewood-Richardson rule and Gelfand-Tsetlin patterns, preprint arXiv:1203.2727.

[DaP] B. A. Davey and H. A. Priestley, Introduction to lattices and order, Second edition, Cambridge University Press, New York, 2002.

[DeP] C. DeConcini and C. Procesi, A characteristic-free approach to invariant theory, Adv. Math. 21 (1976), $330-354$.

[DKR] J. Désarménien, J. Kung, and G.-C. Rota, Invariant theory, Young bitableaux, and combinatorics, Adv. Math. 27 (1978), 63 - 92.

[DRS] P. Doubilet, G.-C. Rota and J. Stein, Foundations of Combinatorial Theory, IX, Stud. in App. Math. 53 (1974), 185 - 216.

[DY] R. Dehy and R. W. T Yu, Degeneration of Schubert varieties of $S L_{n} / B$ to toric varieties, Ann. Inst. Fourier (Grenoble) 51 (2001), no. 6, 1525-1538.

[Fu] W. Fulton, Young Tableaux, Cambridge University Press, Cambridge, UK, 1997.

[GL] N. Gonciulea and V. Lakshmibai, Degenerations of flag and Schubert varieties to toric varieties, Transf. Groups 1 (1996), No. 3, 215-248.

[GT] I. M. Gelfand and M. L. Tsetlin, Finite-dimensional representations of the group of unimodular matrices. (Russian) Doklady Akad. Nauk SSSR (N.S.) 71, (1950). 825-828. English translation: I. M. Gelfand, Collected Papers. Vol II. Edited by S. G. Gindikin, V. W. Guillemin, A. A. Kirillov, B. Kostant, and S. Sternberg. Springer-Verlag, Berlin, 1988, 653-656.

[GW] R. Goodman and N. Wallach, Representations and Invariants of the Classical Groups, Cambridge Univ. Press, 1998.

[Hd] W. Hodge, Some enumerative results in the theory of forms, Proc. Cam. Phi. Soc. 39 (1943), 22-30.

[Hi] T. Hibi, Distributive lattices, affine semigroup rings and algebras with straightening laws, in: Commutative Algebra and Combinatorics, in: Adv. Stud. Pure Math., vol. 11, 1987, pp. 93-109.

[Ho1] R. Howe, Remarks on classical invariant theory, Trans. Amer. Math. Soc. 313 (1989), 539-570. 
[Ho2] R. Howe, Perspectives on invariant theory, The Schur Lectures, I. Piatetski-Shapiro and S. Gelbart (eds.), Israel Mathematical Conference Proceedings, 1995, 1-182.

[Ho3] R. Howe, Weyl Chambers and Standard Monomial Theory for Poset Lattice Cones, Q. J. Pure Appl. Math. 1 (2005) 227-239.

$\left[\mathrm{HJL}^{+}\right]$R. Howe, S. Jackson, S. T. Lee, E-C Tan and J. Willenbring, Toric degeneration of branching algebras, Adv. Math. 220 (2009), no. 6, 1809-1841.

[HL1] R. Howe and S. T. Lee, Bases for some reciprocity algebras I, Trans. Amer. Math. Soc. 359 (2007), 4359-4387.

[HL2] R. Howe and S. T. Lee, Why should the Littlewood-Richardson rule be true?, Bull. Amer. Math. Soc. 43 (2012), 187-236.

[HTW1] R. Howe, E.-C. Tan and J. Willenbring, Reciprocity algebras and branching for classical symmetric pairs, in "Groups and Analysis - the Legacy of Hermann Weyl," London Mathematical Society Lecture Note Series No. 354, Cambridge University Press, 2008, 191-231.

[HTW2] R. Howe, E.-C. Tan and J. Willenbring, Stable branching rules for classical symmetric pairs, Trans. Amer. Math. Soc. 357 (2005) 1601-1626.

[Kas] M. Kashiwara, Crystalizing the q-analogue of universal enveloping algebras, Comm. Math. Phys. 133 (1990), no. 2, 249-260.

[Kav] K. Kaveh, SAGBI bases and degeneration of spherical varieties to toric varieties, Michigan Math. J., 53 (2005), 109-121.

[Ki1] S. Kim, Standard monomial theory for flag algebras of $G L(n)$ and $S p(2 n)$, J. Algebra 320(2) (2008), 534-568.

[Ki2] S. Kim, Standard monomial bases and degenerations of $S_{m}(\mathbb{C})$ representations, J. Algebra 322(11) (2009), 3896-3911.

[Ki3] S. Kim, The nullcone in the multi-vector representation of the symplectic group and related combinatorics, J. Combin. Theory Ser. A 117 (2010), no. 8, 1231-1247.

[Ki4] S. Kim, Distributive lattices, affine semigroups, and branching rules of the classical groups, J. Combin. Theory Ser. A 119 (2012), 1132-1157.

[KL] S. Kim and S. T. Lee, Pieri algebras for the orthogonal and symplectic groups, Israel J. Math., to appear. DOI: 10.1007/s11856-012-0105-1, preprint arXiv:0907.1336.

[KY] S. Kim and O. Yacobi, A basis for the symplectic group branching algebra, J. Algebraic Combin. 35 (2012), no. 2, 269-290.

[Kir] A. A. Kirillov, A remark on the Gelfand-Tsetlin patterns for symplectic groups, J. Geom. Phys. 5 (1988), 473-482.

[Kly] A. A. Klyachko, Stable bundles, representation theory and Hermitian operators, Selecta Math. (N.S.) 4, 1998, no. 3, 419-445.

[KT1] A. Knutson and T. Tao, The honeycomb model of $G L_{n}(\mathbb{C})$ tensor products I: Proof of the saturation conjecture, J. Amer. Math. Soc. 12 (1999), 1055-1090.

[KT2] A. Knutson, T. Tao and C. Woodward, The honeycomb model of $G L_{n}(\mathbb{C})$ tensor products II: Puzzles determine facets of the Littlewood-Richardson cone, J. Amer. Math. Soc. 17 (2003), No. 1, 19-48.

[KM] M. Kogan and E. Miller, Toric degeneration of Schubert varieties and Gelfand-Tsetlin polytopes. Adv. Math. 193 (2005), no. 1, 1-17.

[Ku] S. Kudla, Seesaw dual reductive pairs, in: Automorphic Forms of Several Variables, Taniguchi Symposium, Katata, 1983, Birkhauser, Boston (1983), 244-268.

[Li] P. Littelmann, Paths and root operators in representation theory, Ann. of Math. 142 (1995), no. 3, 499-525.

[LR] D. Littlewood and A. Richardson, Group characters and algebra, Philos. Trans. Roy. Soc. Londo. Ser. A. 233, (1934), 99-142.

[Lu] G. Lusztig, Canonical bases arising from quantized enveloping algebras, J. Amer. Math. Soc. 3 (1990), no. 2, 447-498

[Ma] I. Macdonald, Symmetric Functions and Hall Polynomials, Oxford University Press, 1995.

[MS] E. Miller and B. Sturmfels, Combinatorial Commutative Algebra, Graduate Texts in Mathematics 227, Springer-Verlag, New York, 2005.

[Mo] A. Molev, A basis for representations of symplectic Lie algebras, Comm. Math. Phys. 201 (1999), 591-618.

[PV] I. Pak and E. Vallejo, Combinatorics and geometry of Littlewood-Richardson cones, Europ. J. Combinatorics 26 (2005), 995-1008. 
[Rei] V. Reiner, Signed posets, J. Combin. Theory Ser. A 62 (1993), 324-360.

[RS] J. Remmel and M. Shimozono, A simple proof of the Littlewood-Richardson rule and applications, Discrete Math. 193 (1998), 257-266.

[Sch] M. Schützenberger, La correspondance de Robinson, pp. 59-113 in Combinatoire et représentation du groupe symétrique (D. Foata, ed.), Lecture Notes in Mathematics 579, 1976.

[Sta] R. Stanley, Enumerative combinatorics, Volume 2, Cambridge University Press, 1999.

[Stm] J. Stembridge, A concise proof of the Littlewood-Richardson rule, Electron. J. Combin. 9 (2002), no. 1, Note 5, 4 pp. (electronic).

[Stu] B. Sturmfels, Gröbner Beses and Convex Polytopes, Univ. Lecture Series, Vol. 8, Amer. Math. Soc., Providence, RI, 1996.

[Su1] S. Sundaram, Orthogonal tableaux and an insertion algorithm for $\mathrm{SO}(2 n+1)$. J. Combin. Theory Ser. A 53 (1990), no. 2, 239-256.

[Su2] S. Sundaram, The Cauchy identity for $\operatorname{Sp}(2 n)$. J. Combin. Theory Ser. A 53 (1990), no. 2, 209-238

[Th1] G. P. Thomas, Baxter algebras and Schur functions, Ph. D. thesis, University College of Swansea, (1974).

[Th2] G. P. Thomas, On Schensted's construction and the multiplication of Schur functions, Adv. Math. 30 (1978), 8-32.

[Wy] H. Weyl, The Classical Groups, Princeton University Press, Princeton, N.J., 1946.

[Ze] A. Zelevinsky, A generalisation of the Littlewood-Richardson rule and the Robinson-SchenstedKnuth correspondence, J. of Algebra 69 (1981), 82-94.

[Zh] D. Zhelobenko, On Gelfand-Zetlin bases for classical Lie algebras, in: Kirillov, A. A. (ed.) Representations of Lie groups and Lie algebras, Budapest: Akademiai Kiado: 1985, 79-106.

Department of Mathematics, Yale University, New Haven, CT 06520-8283, USA.

E-mail address: howe@math.yale.edu

Department of Mathematics, Ewha Womans University, Seoul 120-750, South Korea.

E-mail address: sangjib@gmail.com

Department of Mathematics, National University of Singapore, Block S17, 10, Lower Kent Ridge Road, Singapore 119076, Singapore.

E-mail address: matleest@nus.edu.sg 